\title{
Global Prevalence of Post-Acute Sequelae of COVID-19 (PASC) or Long COVID: A Meta-Analysis and Systematic Review
}

\section{Authors:}

Chen Chen, MA ${ }^{1, *}$, Spencer R. Haupert, BS ${ }^{1, *}$, Lauren Zimmermann, BSc ${ }^{1,2}$, Xu Shi, PhD ${ }^{1}$, Lars G. Fritsche, $\mathrm{PhD}^{1,3,4}$, Bhramar Mukherjee, $\mathrm{PhD}{ }^{1,2,3,4,5}$

\section{Affiliations:}

${ }^{1}$ Department of Biostatistics, University of Michigan School of Public Health, Ann Arbor, MI 48109, USA

${ }^{2}$ Center for Precision Health Data Science, University of Michigan, Ann Arbor, MI 48109, USA

${ }^{3}$ Rogel Cancer Center, University of Michigan Medicine, Ann Arbor, MI 48109, USA

${ }^{4}$ Center for Statistical Genetics, University of Michigan School of Public Health, Ann Arbor, MI 48109, USA

${ }^{5}$ Department of Epidemiology, University of Michigan School of Public Health, Ann Arbor, MI 48109, USA

*Equal contributions

\section{Correspondence to:}

Bhramar Mukherjee, PhD

Department of Biostatistics

University of Michigan School of Public Health

1415 Washington Heights

Ann Arbor, Ml 48109.

Email: bhramar@umich.edu 


\section{Abstract}

Importance

As SARS-CoV-2 pervades worldwide, considerable focus has been placed on the longer lasting health effects of the virus on the human host and on the anticipated healthcare needs.

\section{Objective}

The primary aim of this study is to examine the prevalence of post-acute sequelae of COVID-19 (PASC), commonly known as long COVID, across the world and to assess geographic heterogeneities through a systematic review and meta-analysis. A second aim is to provide prevalence estimates for individual symptoms that have been commonly reported as PASC, based on the existing literature.

\section{Data Sources}

PubMed, Embase, and iSearch for preprints from medRxiv, bioRxiv, SSRN, and others, were searched on July 5, 2021 with verification extending to August 12, 2021.

\section{Study Selection}

Studies written in English that consider PASC (indexed as ailments persisting at least 28 days after diagnosis or recovery for SARS-CoV-2 infection) and that examine corresponding prevalence, risk factors, duration, or associated symptoms were included. A total of 40 studies were included with 9 from North America, 1 from South America, 17 from Europe, 11 from Asia, and 2 from other regions.

\section{Data Extraction and Synthesis}

Data extraction was performed and separately cross-validated on the following data elements: title, journal, authors, date of publication, outcomes, and characteristics related to the study sample and study design. Using a random effects framework for meta-analysis with DerSimonian-Laird pooled inverse-variance weighted estimator, we provide an interval estimate of PASC prevalence, globally, and across regions. This meta-analysis considers variation in 
medRxiv preprint doi: https://doi.org/10.1101/2021.11.15.21266377; this version posted November 16, 2021. The copyright holder for this preprint (which was not certified by peer review) is the author/funder, who has granted medRxiv a license to display the preprint in perpetuity.

It is made available under a CC-BY 4.0 International license.

PASC prevalence by hospitalization status during the acute phase of infection, duration of symptoms, and specific symptom categories.

\section{Main Outcomes and Measures}

Prevalence of PASC worldwide and stratified by regions.

\section{Results}

Global estimated pooled PASC prevalence derived from the estimates presented in 29 studies was 0.43 (95\% confidence interval [Cl]: $0.35,0.63)$, with a higher pooled PASC prevalence estimate of $0.57(95 \% \mathrm{Cl}: 0.45,0.68)$, among those hospitalized during the acute phase of infection. Females were estimated to have higher pooled PASC prevalence than males $(0.49$ [95\% Cl: $0.35,0.63]$ versus 0.37 [95\% Cl: 0.24, 0.51], respectively). Regional pooled PASC prevalence estimates in descending order were $0.49(95 \% \mathrm{Cl}: 0.21,0.42)$ for Asia, $0.44(95 \%$ Cl: $0.30,0.59)$ for Europe, and $0.30(95 \% \mathrm{Cl}: 0.32,0.66)$ for North America. Global pooled PASC prevalence for $30,60,90$, and 120 days after index test positive date were estimated to be 0.36 (95\% Cl: $0.25,0.48), 0.24$ (95\% Cl: $0.13,0.39), 0.29$ (95\% Cl: $0.12,0.57)$ and 0.51 (95\% Cl: 0.42, 0.59), respectively. Among commonly reported PASC symptoms, fatigue and dyspnea were reported most frequently, with a prevalence of $0.23(95 \% \mathrm{Cl}: 0.13,0.38)$ and 0.13 (95\% Cl: 0.09, 0.19), respectively.

\section{Conclusions and Relevance}

The findings of this meta-analysis suggest that, worldwide, PASC comprises a significant fraction $(0.43$ [95\% Cl: $0.35,0.63])$ of COVID-19 tested positive cases and more than half of hospitalized COVID-19 cases, based on available literature as of August 12, 2021. Geographic differences appear to exist, as lowest to highest PASC prevalence is observed for North America $(0.30$ [95\% Cl: $0.32,0.66])$ to Asia $(0.49$ [95\% Cl: $0.21,0.42])$. The case-mix across studies, in terms of COVID-19 severity during the acute phase of infection and variation in the clinical definition of PASC, may explain some of these differences. Nonetheless, the health 
medRxiv preprint doi: https://doi.org/10.1101/2021.11.15.21266377; this version posted November 16, 2021. The copyright holder for this preprint (which was not certified by peer review) is the author/funder, who has granted medRxiv a license to display the preprint in perpetuity.

It is made available under a CC-BY 4.0 International license .

effects of COVID-19 appear to be prolonged and can exert marked stress on the healthcare

system, with 237M reported COVID-19 cases worldwide as of October 12, 2021. 
medRxiv preprint doi: https://doi.org/10.1101/2021.11.15.21266377; this version posted November 16, 2021. The copyright holder for this preprint (which was not certified by peer review) is the author/funder, who has granted medRxiv a license to display the preprint in perpetuity. It is made available under a CC-BY 4.0 International license .

\section{Key Points}

\section{Question}

Among those infected with COVID-19, what is the global and regional prevalence of post-acute sequelae COVID-19 (PASC)?

\section{Findings}

Globally, the pooled PASC prevalence estimate was 0.43 , whereas the pooled PASC prevalence estimate for patients who had to be hospitalized due to COVID-19 was 0.57.

Regionally, estimated pooled PASC prevalence from largest to smallest effect size were 0.49 for Asia, 0.44 for Europe, and 0.30 for North America. Global pooled PASC prevalence for 30, 60, 90 , and 120 days after index date were estimated to be $0.36,0.24,0.29$, and 0.51 , respectively. Among commonly reported PASC symptoms, fatigue and dyspnea were reported most frequently, with a prevalence of 0.23 and 0.13 .

\section{Meaning}

In follow-up studies of patients with COVID-19 infections, PASC was common both globally and across geographic regions, with studies from Asia reporting the highest prevalence. 
medRxiv preprint doi: https://doi.org/10.1101/2021.11.15.21266377; this version posted November 16, 2021. The copyright holder for this preprint (which was not certified by peer review) is the author/funder, who has granted medRxiv a license to display the preprint in perpetuity.

It is made available under a CC-BY 4.0 International license.

\section{Introduction}

Coronavirus Disease 2019 (COVID-19), a highly transmissible disease caused by the severe acute respiratory syndrome coronavirus 2 (SARS-CoV-2), has presented extraordinary challenges to the global healthcare system. Fever, dry cough, fatigue, anosmia, and dyspnea are some of the most common symptoms associated with the acute phase of infection. ${ }^{1}$ Additionally, albeit less commonly, symptoms affecting a wide range of organ systems including the brain, kidney, and heart may also accompany a COVID-19 infection. ${ }^{2}$ With respect to the burden of the virus worldwide, there have been over 237 million COVID-19 cases and over 4.8 million deaths, as of October $12,2021 .{ }^{3}$ Although the vast majority of those infected survive with an ensuing estimated case fatality rate of $2 \%$, survivors of COVID-19 are known to be at-risk for a variety of sequelae - a condition known as Post-Acute Sequelae of COVID-19 (PASC). ${ }^{4}$ Further obscuring this picture, there is also a large fraction of covert infections due to a multitude of reasons including asymptomatic infections, ${ }^{5}$ barrier to testing ${ }^{6,7}$ and underreporting..$^{8,9}$ Indeed, a recent, extensive review estimated the worldwide pooled asymptomatic percentage of COVID-19 infections to be $35.1 \%(95 \% \mathrm{Cl}: 30.7$ to $39.9 \%$ ), as of August 2021. ${ }^{10}$ Tying to the former, covert infections need be considered with respect to the scope of the prolonged health effects of COVID-19.

In the literature, the occurrence of long-term ailments of COVID-19 appears in a variety of names including PASC, Long COVID, Post-Acute COVID-19 Syndrome (PACS), Chronic COVID-19, and Long Haul COVID-19. It is commonly defined as new or persistent symptoms at 4 or more weeks from infection with SARS-CoV-2. ${ }^{4}$ Carfi et al. were among the first to report post-COVID-19 complications, finding $87.4 \%$ of hospitalized patients had at least one persistent symptom at a mean of 60.3 days after symptom onset. ${ }^{11}$ Early in 2021 , a large UK-based study found that rates of respiratory disease, diabetes, and cardiovascular disease were $6.0(95 \% \mathrm{Cl}$ : 
medRxiv preprint doi: https://doi.org/10.1101/2021.11.15.21266377; this version posted November 16, 2021. The copyright holder for this preprint (which was not certified by peer review) is the author/funder, who has granted medRxiv a license to display the preprint in perpetuity. It is made available under a CC-BY 4.0 International license.

$5.7,6.2), 1.5(95 \% \mathrm{Cl}: 1.4,1.6)$, and $3.0(95 \% \mathrm{Cl}: 2.7,3.2)$ times higher, respectively, in those with a COVID-19 diagnosis as compared to matched controls at a mean follow-up of 140 days. ${ }^{12}$ A more recent meta-analysis estimated $80 \%$ of those infected with SARS-CoV-2 develop at least one long-term symptom, with the most prevalent symptoms being fatigue, headache, attention disorder, hair loss, and dyspnea. ${ }^{13}$ However, as the meta-analysis was conducted in the earlier stage of the pandemic, the review was limited by the inherently smaller sample size of infected individuals underlying the existing studies at that time. Upon this base, further research was forged into the potential factors that see increased PASC prevalence.

Time since infection, acute phase severity, geographic region, and select sociodemographic characteristics, such as age and sex, are among the constellation of factors likely to influence PASC prevalence estimates. Although a large proportion of the current evidence focuses on the hospitalized COVID-19 population, a German study found $34.8 \%$ of COVID-19 patients, with only a mild acute infection, had PASC at 7 months. ${ }^{14}$ To illustrate the geographic heterogeneity seen in PASC prevalence estimates, specific studies from the USA, Italy, and China report prevalence of $28 \%, 51 \%$, and $76 \%$, respectively. ${ }^{15-17}$ Regarding demographic factors, Sudre et al. found female sex to be associated with developing PASC. ${ }^{18}$ Although no existing global reviews (at the time of this report) present age-specific PASC prevalence, Nasserie et al. (2021) offer some evidence that prolonged symptoms are not distinct to older versus younger age groups. ${ }^{19}$ That is, although the bulk of PASC exhibiting individuals across the included studies were older (median age near 60 years), younger age groups were also found to comprise a non-negligible number of those with persistent symptoms. ${ }^{19}$ Existing research suggests older age to be associated with a moderate increased risk of persisting symptoms (for ten-year increments past age of 40 , estimated odds ratio (OR) is 1.10 [95\% Cl: $1.01-1.19]) .{ }^{20}$ Moreover, the existing inequities by race/ethnicity as it pertains to PASC remain largely unexplored, ${ }^{21}$ despite the same having been shown for COVID-19. Select comorbidities have been identified 
medRxiv preprint doi: https://doi.org/10.1101/2021.11.15.21266377; this version posted November 16, 2021. The copyright holder for this preprint (which was not certified by peer review) is the author/funder, who has granted medRxiv a license to display the preprint in perpetuity.

It is made available under a CC-BY 4.0 International license.

as being associated with PASC in the existent literature (e.g., increased risk of PASC among individuals with asthma, ${ }^{18}$ though these findings are generally in early stages).

Following these collective efforts, we too emphasize that PASC must be well-defined and wellunderstood to enable data to inform clinical decision-making and guidance, and thereby, aid the millions of affected individuals worldwide. At this juncture of being nearly two years into the COVID-19 pandemic, numerous large, high-quality studies on PASC, with substantial follow-up time, have been conducted. Expanding on previous meta-analyses hampered by smaller sample sizes and shorter follow-up times, this systematic review and meta-analysis aims to provide a comprehensive synthesis of information on prevalence and symptoms of PASC todate. Based on previous research, we hypothesize that PASC is common across geographic and demographic groups, with respiratory, neurological, cardiac, and psychological symptoms having the highest prevalence. We close with some avenues for future research considerations, as highlighted by the findings of this review.

\section{Methods}

\section{Search Strategy}

We employed PICO and PRISMA frameworks to guide our entire research process (eTable 1). ${ }^{22}$ The literature databases, PubMed and Embase for published articles, as well as iSearch for preprint articles from bioRxiv, medRxiv, SSRN, Research Square, and preprints.org, were searched on July 5, 2021, and search verification was extended through August 12,2021 . The search aimed to capture papers relating to PASC and that examine prevalence, risk factors, and/or duration, published during the years 2020-2021, and written in English. We adapted some search components from a public resource made available by Yale University Libraries. ${ }^{23}$ The full search strategy, including filters for each database, is presented in eMethods 1. 


\section{Screening Procedure}

A two-step approach to screening was used with an initial title/abstract screening, followed by a full-text screening, and an ultimate discussion and re-examination to resolve conflicting marks. Screeners 1 and 2 performed both phases of the screening independently (i.e., were blinded). Rayyan, a web-based application, was used as a tool to help expedite literature screening for systematic reviews. ${ }^{24}$

Our inclusion criteria were as follows: (1) human study population with confirmed COVID-19 diagnosis through PCR test, antibody test, or diagnosis, (2) index date of first test/diagnosis, date of hospitalization, discharge date, or date of clinical recovery/negative test, (3) primary outcome must include prevalence, risk factors, duration, or symptoms of PASC, and (4) the follow-up time is at least 28 days after the index date. We excluded case studies, reviews, studies with imaging or molecular/cellular testing as primary results, and studies with only healthcare workers or residents of nursing homes/long-term care facilities. We also excluded studies that did not meet the sample size threshold of 323 , pre-calculated herein. The reason for this is to ensure the included studies were adequately powered to achieve a margin of error of at least 0.05 on the provided PASC prevalence estimate. The sample size threshold was calculated with an estimated prevalence of $30 \%$ and for a $95 \%$ Wald-type confidence interval for binomial proportion; see eMethods 2 for further details.

\section{Data Extraction}

After studies were selected, the following relevant data elements were manually extracted separately by both screeners 1 and 2: article title, authors, date of publication, study purpose, study design, population, setting, country, sample size, method of COVID-19 confirmation, index date, follow-up time, demographic variables (i.e., age and sex), and outcomes examined. In the 
medRxiv preprint doi: https://doi.org/10.1101/2021.11.15.21266377; this version posted November 16, 2021. The copyright holder for this preprint (which was not certified by peer review) is the author/funder, who has granted medRxiv a license to display the preprint in perpetuity.

It is made available under a CC-BY 4.0 International license.

instance of multiple study versions with the same underlying population, we used the most recently published article.

\section{Outcomes and measures}

The primary outcome was the prevalence of PASC and symptoms at least 28 days after the index date. We defined PASC as having any symptoms, or at least one new or persisting symptom during the follow-up time. The follow-up time of COVID-19 patients across studies was divided into the following four groups: PASC persisting at 28-30 days (labeled as 30 days), 60 days, 90 days, and 120 days after the index date. We combined similar symptoms into a broader concept. For example, we joined together dyspnea, shortness of breath, and problem of breathing reported in different studies into a broader symptom concept of dyspnea (see eTable

2). Studies were classified into the following three groups based on the study population of PASC: (1) studies with non-hospitalized COVID-19 positive individuals, (2) studies with hospitalized COVID-19 positive individuals, (3) studies with all COVID-19 positive individuals (i.e., a case-mix with hospitalized and non-hospitalized individuals). In addition to prevalence, we were also interested in the risk factors for PASC as secondary outcomes.

\section{Statistical Analysis}

Meta-analysis with random effects and generic inverse variance weighting was performed to estimate the prevalence of PASC and symptoms, for outcomes reported in at least five studies. Of further note is that upon examining the distribution of PASC prevalence, we apply a logit transformation to the proportion. The confidence interval was calculated incorporating betweenstudy variance obtained by the DerSimonian-Laird (DL) estimator (eMethods 3). Heterogeneity among studies was reflected by the $I^{2}$ statistic, where $I^{2}$ between $75 \%$ and $100 \%$ indicates considerable heterogeneity. We further stratified our analysis by (1) study population type (hospitalized versus mixed hospitalized and non-hospitalized), (2) sex (female versus male), (3) 
follow-up time, (4) region (Asia, Europe, and USA). Another stratified analysis is presented in the supplement (see eFigure 1) wherein pooled PASC prevalence is estimated (A) among studies defining PASC to be persisting symptoms (i.e., extended beyond a pre-specified number of days) and (B) among studies defining PASC to be at least one symptom or not recovered from COVID-19. All analyses were conducted in $\mathrm{R}$ (version 4.0.2) using packages meta ${ }^{25,26}$ and metafor. ${ }^{27}$

For critical appraisal, we used a checklist-based tool from Joanna Briggs Institute (JBI), corresponding to prevalence studies and hence, enabling assessment of risk of bias among the included study designs. ${ }^{28}$ Assessment of publication bias was carried out visually by generating funnel plot and formally by conducting Egger's and Begg's tests for funnel plot asymmetry (for further details, see eMethods 4 and eFigure 2).

\section{Results}

\section{Search Results}

In our main literature search, we identified 4,438 unique citations of which 270 had titles or abstracts that passed our criteria for a full-text assessment. After the full-text screen, we deemed 40 studies eligible for a qualitative synthesis, of which we further meta-analyzed reported measures from 34 with compatible outcomes. See the PRISMA flow diagram (Figure 1) and eTable 3 for details concerning study inclusion/exclusion criteria. In efforts to further verify the search results, we performed a second literature search one month after the first screen, although no additional eligible studies were identified (eMethods 1 and eFigure 3).

\section{Study Characteristics}


medRxiv preprint doi: https://doi.org/10.1101/2021.11.15.21266377; this version posted November 16, 2021. The copyright holder for this preprint (which was not certified by peer review) is the author/funder, who has granted medRxiv a license to display the preprint in perpetuity.

It is made available under a CC-BY 4.0 International license .

Table 1 shows the characteristics of all 40 included articles. The studies comprised a total of 886,388 COVID-19 positive patients that we categorized into non-hospitalized (3,371 patients from 4 studies), hospitalized (61,247 patients from 17 studies), and any COVID-19 positive patients regardless of severity or hospitalization status (821,770 individuals from 19 studies). While only studies with at least 4 weeks follow-up were selected, several studies had data on substantially longer follow-up times: for at least 8 weeks (36 studies), 12 weeks (33 studies), and 6 months (17 studies). Figure 1 lists additional study characteristics.

\section{PASC Prevalence}

Among the 34 included studies in the quantitative synthesis, we meta-analyzed the 29 studies reporting an overall prevalence of PASC. Pooled global PASC prevalence was estimated to be $0.43(95 \% \mathrm{Cl}: 0.35,0.63)$ (Table 2). Substantial heterogeneity was observed among the included studies $\left(I^{2}=100 \%, P<0.001\right)$. Estimates ranged widely from 0.09 to 0.81 which may in part be driven by differences in terms of sex, region, COVID-19 study population, and followup time. For example, the studies that included only hospitalized cases tended to show higher PASC prevalence than non-hospitalized or the mix of hospitalized and non-hospitalized patients (Figure 2). To better understand the interplay of these factors with PASC prevalence estimates, we performed additional stratified meta-analyses (Table 2).

First, the pooled PASC prevalence in hospitalized patients of $0.57(95 \% \mathrm{Cl}: 0.45,0.68)$ compared to the estimate in a mix of hospitalized and non-hospitalized COVID-19 patients of $0.31(95 \% \mathrm{Cl}: 0.24,0.40)$ revealed a sizeable difference, further distinguished by nonoverlapping confidence intervals. However, we note that a wide range of estimates contributed to both groups (i.e., PASC prevalence varied from $0.25-0.81$ in hospitalized studies versus $0.09-0.62$ in the mixed group, and significant heterogeneity was present in both) (eFigure 4B). 
medRxiv preprint doi: https://doi.org/10.1101/2021.11.15.21266377; this version posted November 16, 2021. The copyright holder for this preprint (which was not certified by peer review) is the author/funder, who has granted medRxiv a license to display the preprint in perpetuity.

It is made available under a CC-BY 4.0 International license .

Next, when focusing on sex, we estimated a pooled PASC prevalence in females of $0.49(95 \%$ $\mathrm{Cl}: 0.35,0.63)$, which was higher than that in males of $0.37(95 \% \mathrm{Cl}: 0.24,0.51)$. Considering the same studies underly both strata, this imbalance was unlikely attributable to differences in the contributing studies (eFigure 4A).

Examining region-specific prevalences, pooled estimated prevalence of PASC was lower in the USA at $0.30(95 \% \mathrm{Cl}: 0.21,0.42)$ than in Europe at $0.43(95 \% \mathrm{Cl}: 0.29,0.58)$, while the highest estimated prevalence was in Asia at 0.49 (95\% Cl: 0.32, 0.66). Considerable within-region variation was observed among the included studies in that the corresponding ranges of prevalence of PASC were generally wide, with Europe exhibiting the largest range of $0.09-$ 0.81. Overall, we did not identify any patterns with respect to particular countries that could explain the heterogeneity within each of the meta-analyzed regions $(P<0.001$; eFigure $4 \mathrm{C})$.

Finally, we focused on estimating PASC prevalence stratified by follow-up time. With increasing follow-up time from 30 to 60 days after the index date, the estimated pooled prevalence of PASC decreased from $0.36(95 \% \mathrm{Cl}: 0.25,0.48)$ to $0.24(95 \% \mathrm{Cl}: 0.13,0.39)$. Pooled prevalence of PASC 90 and 120 days after the index date further increased to 0.29 (95\% Cl: $0.12,0.57)$ and to $0.51(95 \% \mathrm{Cl}: 0.42,0.59)$, respectively (eFigure 4D). A possible reason for this comparatively high PASC prevalence at 120 days of follow-up time is that the bulk of the studies underlying this estimate concentrated on hospitalized populations (eFigure 5). Studies are also likely to experience higher drop-out rates as follow-up time increases, resulting in individuals no longer experiencing symptoms being underrepresented at the later time points. Some studies that measured PASC prevalence at multiple time points experienced a similar phenomenon. ${ }^{14}$ 
medRxiv preprint doi: https://doi.org/10.1101/2021.11.15.21266377; this version posted November 16, 2021. The copyright holder for this preprint (which was not certified by peer review) is the author/funder, who has granted medRxiv a license to display the preprint in perpetuity.

It is made available under a CC-BY 4.0 International license.

Significant levels of heterogeneity being present within each stratified meta-analysis corroborates that no single factor alone may account for the variation in PASC prevalence, but that rather a combination of factors should be considered. Concerning a perceived ordering of influence, the factors that seem to have the largest bearing on the PASC prevalence (as ordered from highest to lowest) were study population, region, and follow-up time. Furthermore, inconsistent PASC definition is a source of heterogeneity. As detailed in eFigure 1, studies measuring PASC as having at least one persistent symptom had lower heterogeneity (as measured by a chi-square test statistic) compared to those measuring PASC as at least one symptom (with symptoms not necessarily starting during the acute phase). Noting that the prevalence of each symptom varied, effect size of PASC prevalence estimates may differ in part due to the underlying symptoms assessed therein. An additional meta-analysis of studies with at least 120 days follow-up stratified by COVID-19 population resulted in similar observed heterogeneity (eFigure 5). Ultimately, these findings suggest that such variation may be indelible, as key considerations, such as the definition of PASC itself, as well as other clinical and methodological subcomponents, remain largely in flux. ${ }^{62}$

\section{Prevalence of specific PASC symptoms}

Considering a unified definition of PASC remains under investigation (as discussed in the Introduction section), it was important to understand the prevalence of specific symptoms after COVID-19. In total, we assessed 23 symptoms reported across 30 studies (Table 2, Figure 3). The five most prevalent symptoms were the following, with corresponding estimated pooled symptom-specific prevalence: fatigue at $0.23(95 \% \mathrm{Cl}: 0.13,0.38)$, dyspnea at $0.13(95 \% \mathrm{Cl}$ : $0.09,0.19)$, insomnia at $0.13(95 \% \mathrm{Cl}: 0.06,0.28)$, joint pain at $0.13(95 \% \mathrm{Cl}: 0.05,0.29)$, and memory problems at $0.13(95 \% \mathrm{Cl}: 0.10,0.18)$. Forest plots for symptom-specific prevalence estimates are presented in eFigure $\mathbf{6}$. We note that a study by Orrū et al. from Italy tended to fall toward the higher end of the observed range for several symptom categories, and as such 
medRxiv preprint doi: https://doi.org/10.1101/2021.11.15.21266377; this version posted November 16, 2021. The copyright holder for this preprint (which was not certified by peer review) is the author/funder, who has granted medRxiv a license to display the preprint in perpetuity.

It is made available under a CC-BY 4.0 International license.

this outlying study (relative to the other underlying studies) may have skewed the resulting point estimates and confidence intervals to a degree. ${ }^{43}$

\section{PASC risk factors}

Although all included studies were screened for reported PASC risk factors, sex and preexisting asthma were the only risk factors that were estimated in multiple studies and thus metaanalyzed. Female sex and pre-existing asthma had higher odds of having PASC with pooled estimated odds ratios (OR) of 1.57 (95\% Cl: 1.09, 2.26) and 2.15 (95\% Cl: 1.14, 4.05), respectively. Both meta-analyzed ORs were based on less than 5 studies and should thus be interpreted with caution. Among the studies that were not meta-analyzed, several found that individuals with more severe COVID-19 during the acute phase had higher risk of developing PASC. ${ }^{37,45,50,52}$ Additionally, two studies found older age to be associated with PASC. ${ }^{20,58}$ Other risk factors for PASC including number of symptoms during acute COVID- $19,{ }^{16}$ fatigue ${ }^{18}$, dyspnea, ${ }^{18,39}$ muscle pain, ${ }^{50}$ headache,${ }^{18,20}$ myalgia, ${ }^{18}$ and pre-existing conditions such as obesity, ${ }^{18,52}$ comorbidity, ${ }^{45}$ and hypothyroidism ${ }^{37}$ were found positively associated with PASC (eTable 4).

\section{Systematic review}

Six studies were not included in the meta-analysis since they did not report a composite binary endpoint as prevalence (Figure 1). Three studies used incidence rate or incidence density to measure PASC. Chevinsky et al. reported a $7 \%$ incidence rate of at least one of the five most common new conditions during days 31 to 120 for inpatients and a $7.7 \%$ incidence rate for at least one of 10 new conditions. ${ }^{57}$ A UK study found breathlessness (85 and 536 events per 100,000 person-years in non-hospitalized and hospitalized patients) and joint pain (168 and 295 events per 100,000 person-years in non-hospitalized and hospitalized patients) to be the most common sequelae at 2 months. ${ }^{52}$ Another UK study found the rates of respiratory disease and 
medRxiv preprint doi: https://doi.org/10.1101/2021.11.15.21266377; this version posted November 16, 2021. The copyright holder for this preprint (which was not certified by peer review) is the author/funder, who has granted medRxiv a license to display the preprint in perpetuity.

It is made available under a CC-BY 4.0 International license .

major cardiovascular events to be $770.5(95 \% \mathrm{Cl}: 757.8,783.3)$ and $126(95 \% \mathrm{Cl}: 121,131)$ events per 1,000 person-years. ${ }^{12}$ The other three studies investigated PASC with a focus on the psychiatric and neurological illness. Damiano et al. focused on psychiatric and cognitive sequela and reported a prevalence of $0.08(95 \% \mathrm{Cl}: 0.06,0.11), 0.14(95 \% \mathrm{Cl}: 0.11,0.18)$, and 0.16 $(95 \% \mathrm{Cl}: 0.12,19)$ for depression, generalized anxiety disorder, and mixed anxietydepression. ${ }^{56}$ Another study by Taquet et al. also concentrated on psychiatric disorder and measured incidence and hazard ratio of psychiatric disorder, dementia and insomnia with 90 days follow up. ${ }^{61}$ The estimated probability of having new psychiatric illness 90 days after COVID-19 diagnosis was 5.8\% (95\% Cl: 5.2, 6.4) Huang et al.'s study from China showed psychosocial problems (57.7\%), worse depression (35\%), and worse dyspnea (32.6\%) to be among the most common complaints $4-6$ months after discharge. ${ }^{29}$

Two articles described the duration of PASC and persistent symptoms. According to Sudre et al., the median duration of PASC with persisting symptoms was 41 days. For persisting symptoms that occurred at least 28 days after COVID-19 diagnosis, the median duration of persisting fatigue, headache, dyspnea, and myalgia was 33 days, 22 days, 24 days, and 7 days, respectively. ${ }^{18}$ However, the median duration of persisting symptoms was longer in another India study. ${ }^{35} \mathrm{~A}$ summary table of duration was reported in eTable 5.

\section{Discussion}

We screened nearly 4.5 thousand articles and synthesized information from 40 large studies including almost one million individuals worldwide. The empirical findings suggest a global PASC prevalence of approximately $43 \%$. Based on a WHO estimate of 237 million worldwide COVID-19 infections, this global pooled PASC estimate indicates that around 100 million individuals currently experience or have previously experienced long-term health-related 
medRxiv preprint doi: https://doi.org/10.1101/2021.11.15.21266377; this version posted November 16, 2021. The copyright holder for this preprint (which was not certified by peer review) is the author/funder, who has granted medRxiv a license to display the preprint in perpetuity.

It is made available under a CC-BY 4.0 International license .

consequences of COVID-19. Individuals who were hospitalized during acute COVID-19 infection had higher PASC prevalence at $57 \%$. Female adults had both higher prevalence and risk of having PASC than male adults (49\% vs $37 \%$ ). The prevalence of PASC in Asia, Europe, and USA are approximately $49 \%, 43 \%$, and $30 \%$, respectively. Next, we contextualize our results among findings from other PASC-related reviews.

Our global PASC estimate of $43 \%$ is considerably lower than the $80 \%$ figure provided by LopezLeon et al. ${ }^{63}$ Their most prevalent sequela was fatigue at $58 \%$ which is concordant with fatigue being the most prevalent sequela at $23 \%$ in this study. In general, empirical symptom-specific prevalence estimates are lower in this study, although multiple estimates (e.g., for insomnia, memory problems, anxiety, depression) generally reconcile with the Lopez-Leon et al. review. ${ }^{13}$ Similarly, when comparing to the lqbal et al. ${ }^{64}$ meta-analyzed PASC-related symptom prevalence findings, the estimates herein are lower. A potential reasoning for this is the sample size threshold that we employed may have led to select studies being excluded that were conducted in early 2020 with smaller samples and focused mainly on sicker patients.

Additional notable studies have been published after the date of this systematic search (August 12, 2021), and as such are not captured in the empirical estimates presented herein. As examples, another study by Taquet et al., using the TriNetX Analytics EHR network, estimated $36.55 \%$ of COVID-19 patients to have at least one PASC-related symptom 3-6 months after diagnosis. ${ }^{65}$ Huang et al. 2021 also provided an update on their cohort from Jin Yin-tan Hospital in Wuhan. ${ }^{66}$ Their 6-month study (which is included in this review) estimated 6-month PASC to be $76 \%$. Their 12-month update found that, among patients who attended both the 6-month and 12-month follow-up, PASC prevalence decreased from $68 \%$ at 6 months to $49 \%$ at 12 months. 
medRxiv preprint doi: https://doi.org/10.1101/2021.11.15.21266377; this version posted November 16, 2021. The copyright holder for this preprint (which was not certified by peer review) is the author/funder, who has granted medRxiv a license to display the preprint in perpetuity. It is made available under a CC-BY 4.0 International license.

In addition to experiencing PASC symptoms, some COVID-19 survivors also go on to develop other complications. For the purposes of this review, we define COVID-19 complication as any secondary disease that manifests after the acute phase of a COVID-19 infection. Multisystem Inflammatory Syndrome in Children (MIS-C), Chronic Kidney Disease (CKD), myocarditis/pericarditis, Chronic Fatigue Syndrome (CFS) or myalgic encephalomyelitis, and Kawasaki disease are complications known to be associated with COVID-19. ${ }^{67}$ While the focus of this review is on PASC symptoms rather than complications, further research is necessary to understand the relationship between COVID-19 and these complications and the needs of those living with complications.

Our meta-analysis showed that female sex and pre-existing asthma correspond with higher proportions of PASC development. Outside of meta-analysis, we also found age, acute phase symptoms and severity, hypothyroidism, obesity, hypertension, and other pre-existing conditions to be risk factors for PASC. Protective factors for PASC may also exist, as a recent study suggested vaccines may offer protection. ${ }^{68}$ However, a large hospital-based study suggests the opposite. ${ }^{69}$ As such, the interplay between COVID-19 vaccines and PASC is at-large yet to be determined. Multiple other risk factors for PASC have been detected, and, although encompassed among select included studies, such factors were not meta-analyzed because they did not reach the threshold of at least 5 studies. Increased number of acute-phase symptoms is associated with PASC; however, one study reported $32 \%$ of individuals with PASC were asymptomatic during the acute phase in a non-hospitalized population. ${ }^{55}$ Similarly, few studies examined the duration of PASC. Future research needs to further explore risk factors and duration for PASC, as these are generally critical components for clinicians in screening patients for increased risk of developing PASC, and in devising an appropriate treatment protocol accordingly. This leads to the several limitations of this systematic review and metaanalysis. 
medRxiv preprint doi: https://doi.org/10.1101/2021.11.15.21266377; this version posted November 16, 2021. The copyright holder for this preprint (which was not certified by peer review) is the author/funder, who has granted medRxiv a license to display the preprint in perpetuity.

It is made available under a CC-BY 4.0 International license.

\section{Limitations}

First, we did not survey grey literature (literature not published in academic journals), which could make our results reflect the positive publication bias known to exist in peer-reviewed medical literature, though we included preprints. ${ }^{70}$ Second, we only considered studies written in English which may have excluded important studies written in other languages. Third, while our criteria for follow-up time and index date seem reasonable, there may be important results from studies using other criteria. For example, a large Danish cohort analyzed by Lund et al. was excluded for their choice of follow-up time. ${ }^{71}$ Fourth, bias in testing for COVID-19, especially in the early stages of the pandemic, might have affected the characteristics of the COVID-19 positive cohort. ${ }^{72}$ In other words, patients without access to testing, patients without strong health-seeking behavior, and asymptomatic individuals are not blanketly reflected in the empirical findings. Additionally, included studies conducted in early 2020 may tend to be older and higher risk individuals, as testing among these groups was prioritized at that time. Fifth, our sample size criteria may have curtailed inclusion of early-pandemic studies, as sample sizes were generally smaller at that time, and thus favored studies examining acute-phase manifestations over studies focusing on PASC. Lastly, while our review included studies across 17+ countries, data from multiple regions are largely absent (notably Africa and Australia).

Existing inequities in healthcare access may hamper underserved populations being adequately reflected herein. Moreover, we emphasize that stratifying PASC by race-ethnicity is a noteworthy gap in the literature. With respect to the age composition of the included articles, few children were included in the underlying sample. Future investigators may seek to further examine differences in PASC prevalence among such demographic subgroups.

\section{Conclusions}


medRxiv preprint doi: https://doi.org/10.1101/2021.11.15.21266377; this version posted November 16, 2021. The copyright holder for this preprint (which was not certified by peer review) is the author/funder, who has granted medRxiv a license to display the preprint in perpetuity. It is made available under a CC-BY 4.0 International license.

Findings from this study provide insight into the empirical estimates of prevalence, symptoms, risk factors, and duration of PASC, with an examination of differences by several factors including geography. We recommend continued attention be focused on identifying patients atrisk of developing PASC and on quantifying duration of PASC to aid in the clinical advancements globally for alleviating the long-lasting health effects of COVID-19. 
medRxiv preprint doi: https://doi.org/10.1101/2021.11.15.21266377; this version posted November 16, 2021. The copyright holder for this preprint (which was not certified by peer review) is the author/funder, who has granted medRxiv a license to display the preprint in perpetuity.

It is made available under a CC-BY 4.0 International license .

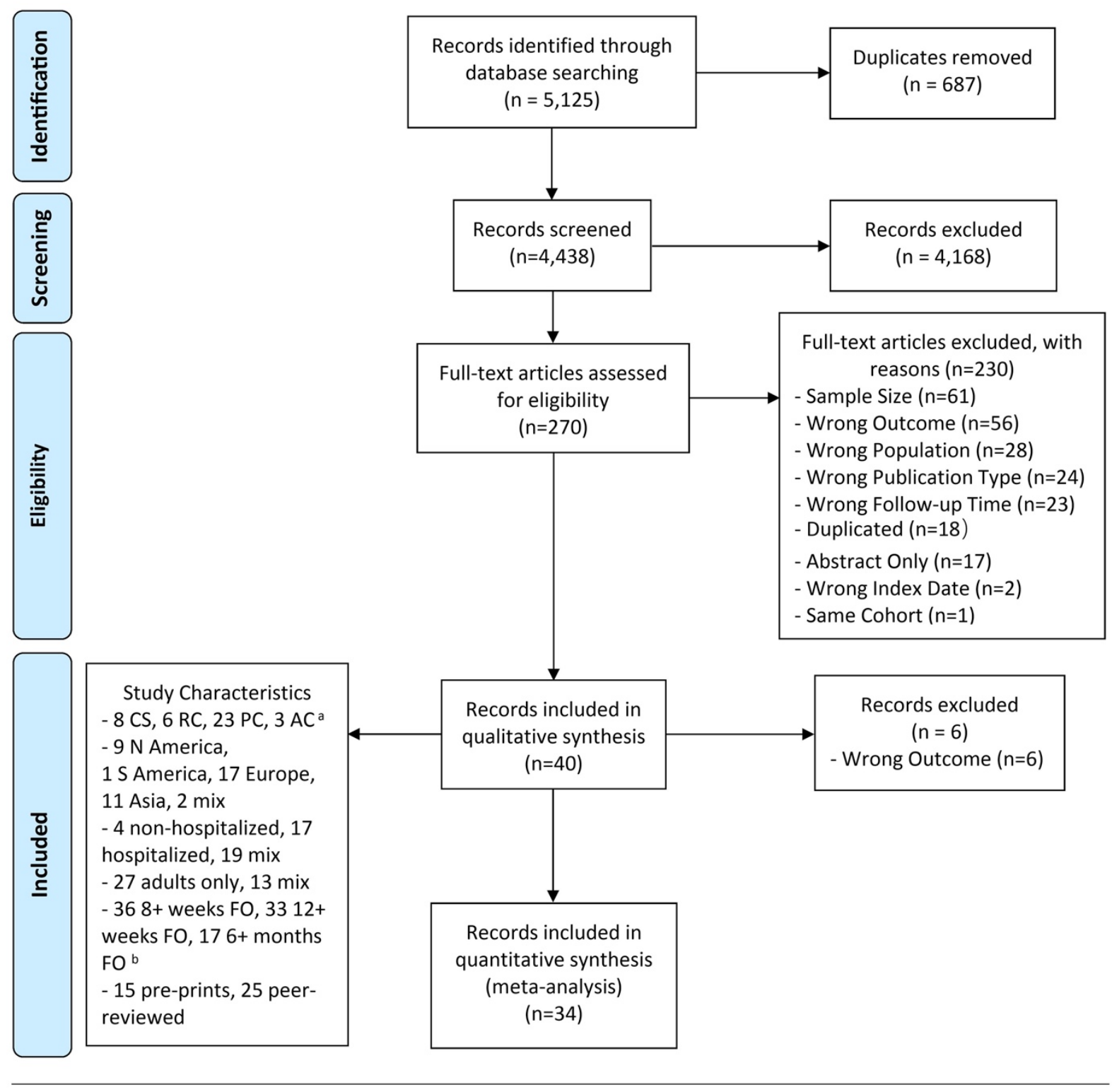

${ }^{\mathrm{a}} \mathrm{CS}=$ cross-sectional, $\mathrm{RC}=$ retrospective cohort, $\mathrm{PC}=$ prospective cohort, $\mathrm{AC}=$ ambidirectional cohort ${ }^{\mathrm{b}} \mathrm{FO}=$ follow-up

Figure 1. PRISMA flow diagram.

Note: Additional study characteristics of all included studies are listed in the box in the bottom left. 
Table 1. Summary of Included Studies.

\begin{tabular}{|c|c|c|c|c|c|c|c|c|c|c|c|}
\hline 응 & $\begin{array}{l}\text { Date of } \\
\text { Publication }\end{array}$ & Authors & $\begin{array}{l}\text { Study } \\
\text { Design } \\
\text { a }\end{array}$ & $\begin{array}{l}\text { Population of } \\
\text { Interest }\end{array}$ & Setting & Country & Sample Size & $\begin{array}{l}\text { Follow-up } \\
\text { Time } f\end{array}$ & Age & $\begin{array}{l}\text { Sex }(\% \\
\text { female) }\end{array}$ & $\begin{array}{l}\text { Outcomes of } \\
\text { Interest } \mathrm{e}\end{array}$ \\
\hline \multirow{11}{*}{$\frac{\pi}{4}$} & Dec 2020 & Huang et $\mathrm{al}^{29}$ & $A C$ & $\begin{array}{l}\text { COVID-19+, } \\
\text { hospitalized } \\
\text { adults }\end{array}$ & $\begin{array}{l}\text { Leishenshan Hospital } \\
\text { (Wuhan) }\end{array}$ & China & 464 & $\begin{array}{l}4-6 \text { months } \\
* \star *\end{array}$ & $57(15-93)^{c}$ & $48.50 \%$ & $\begin{array}{l}\text { Symptom } \\
\text { prevalence, risk } \\
\text { factors }\end{array}$ \\
\hline & Jan 2021 & Huang et $\mathrm{al}^{17}$ & $A C$ & $\begin{array}{l}\text { COVID-19+ } \\
\text { adults }\end{array}$ & $\begin{array}{l}\text { Jin Yin-tan Hospital } \\
\text { (Wuhan) }\end{array}$ & China & 1733 & $\begin{array}{l}186(175- \\
199) \text { days } c \\
* \star \star \star\end{array}$ & $57(47-65)^{c}$ & $48 \%$ & $\begin{array}{l}\text { PASC and } \\
\text { symptom } \\
\text { prevalence }\end{array}$ \\
\hline & Jan 2021 & Xiong et $\mathrm{a}^{30}$ & $\mathrm{PC}$ & $\begin{array}{l}\text { COVID-19+, } \\
\text { hospitalized } \\
\text { adults }\end{array}$ & $\begin{array}{l}\text { Renmin Hospital of } \\
\text { Wuhan University }\end{array}$ & China & 538 & $\begin{array}{l}97(95-102) \\
\text { days }{ }^{* * * *}\end{array}$ & $52(41-62)^{c}$ & $54.50 \%$ & $\begin{array}{l}\text { PASC and } \\
\text { symptom } \\
\text { prevalence }\end{array}$ \\
\hline & Jan 2021 & Zheng et $\mathrm{al}^{31}$ & CS & $\begin{array}{l}\text { COVID-19+, } \\
\text { hospitalized } \\
\text { adults }\end{array}$ & $\begin{array}{l}\text { Multicenter (hospitals in } \\
\text { Wuhan) }\end{array}$ & China & 574 & $\begin{array}{l}241.79 \\
(16.16) \\
\text { days } d^{* * * *}\end{array}$ & $57.7(11.4)^{d}$ & $60.60 \%$ & $\begin{array}{l}\text { Symptom } \\
\text { prevalence }\end{array}$ \\
\hline & Apr 2021 & Shang et $\mathrm{al}^{32}$ & PC & $\begin{array}{l}\text { COVID-19+, } \\
\text { severe, } \\
\text { hospitalized }\end{array}$ & $\begin{array}{l}\text { Multicenter ( } 3 \text { hospitals in } \\
\text { Wuhan) }\end{array}$ & China & 796 & 6 months & $62(51-69)^{c}$ & $49.20 \%$ & $\begin{array}{l}\text { PASC and } \\
\text { symptom } \\
\text { prevalence, risk } \\
\text { factors }\end{array}$ \\
\hline & June 2021 & $\begin{array}{l}\text { Areekal et } \\
\mathrm{al}^{33}\end{array}$ & CS & $\begin{array}{l}\text { COVID-19+, } \\
\text { hospitalized, } \\
\text { symptomatic } \\
\text { adults }\end{array}$ & $\begin{array}{l}\text { Government Medical } \\
\text { College, Thrissur (Kerala) }\end{array}$ & India & 335 & 28 days * & $50.7(15.7)^{d}$ & $48.10 \%$ & $\begin{array}{l}\text { PASC and } \\
\text { symptom } \\
\text { prevalence, risk } \\
\text { factors }\end{array}$ \\
\hline & June 2021 & $\begin{array}{l}\text { Budhiraja et } \\
\text { al }^{34}\end{array}$ & PC & $\begin{array}{l}\text { COVID-19+, } \\
\text { hospitalized }\end{array}$ & $\begin{array}{l}\text { Multicenter (3 Hospitals in } \\
\text { North India) }\end{array}$ & India & 990 & $\begin{array}{l}9(4-12) \\
\text { months d } \\
* \star \star \star\end{array}$ & $\begin{array}{l}14.6 \%<=29 \\
59.7 \% 30-59 \\
25.7 \% 60+\end{array}$ & $67.70 \%$ & $\begin{array}{l}\text { PASC and } \\
\text { symptom } \\
\text { prevalence }\end{array}$ \\
\hline & July 2021 & Naik et al ${ }^{35}$ & PC & $\begin{array}{l}\text { COVID-19+ } \\
\text { adults }\end{array}$ & $\begin{array}{l}\text { Tertiary Care Facility in } \\
\text { New Delhi }\end{array}$ & India & 1,234 & $\begin{array}{l}91(45-185) \\
\text { days }{ }^{* * *}\end{array}$ & $41.4(14.2)^{d}$ & $30.60 \%$ & $\begin{array}{l}\text { PASC and } \\
\text { symptom } \\
\text { prevalence, risk } \\
\text { factors, duration }\end{array}$ \\
\hline & Mar 2021 & $\begin{array}{l}\text { Mannan et } \\
\text { al }^{36}\end{array}$ & CS & $\begin{array}{l}\text { COVID-19+, } \\
\text { hospitalized }\end{array}$ & Multicenter (6 hospitals) & Bangladesh & 1,021 & $4+$ Weeks * & $\begin{array}{l}1.8 \% 0-9 \\
4.9 \% 10-19 \\
24.4 \% 20-29 \\
30.4 \% 30-39 \\
16.8 \% 40-49 \\
12.4 \% 50-59 \\
9.4 \% 60+ \\
\end{array}$ & $25 \%$ & $\begin{array}{l}\text { Symptom } \\
\text { prevalence }\end{array}$ \\
\hline & Nov 2020 & Sami et $\mathrm{al}^{37}$ & $\mathrm{PC}$ & $\begin{array}{l}\text { COVID-19+, } \\
\text { hospitalized } \\
\text { adults }\end{array}$ & $\begin{array}{l}\text { Khorshid Hospital } \\
\text { (Isfahan) }\end{array}$ & Iran & 452 & 4 weeks * & $\mathrm{n} / \mathrm{a}$ & $\mathrm{n} / \mathrm{a}$ & $\begin{array}{l}\text { Symptom } \\
\text { prevalence }\end{array}$ \\
\hline & Aug 2021 & $\begin{array}{l}\text { Munblit et } \\
\mathrm{al}^{38}\end{array}$ & PC & $\begin{array}{l}\text { COVID-19+, } \\
\text { hospitalized } \\
\text { adults }\end{array}$ & $\begin{array}{l}\text { Multicenter (Sechenov } \\
\text { University Hospital } \\
\text { Network, Moscow) }\end{array}$ & Russia & 2,649 & $\begin{array}{l}218(200- \\
236) \text { days c } \\
* * * *\end{array}$ & $56(46-66)^{c}$ & $51.10 \%$ & $\begin{array}{l}\text { PASC and } \\
\text { symptom } \\
\text { prevalence, risk } \\
\text { factors }\end{array}$ \\
\hline \multirow{4}{*}{ ๕ั } & Jan 2021 & $\begin{array}{l}\text { Venturelli et } \\
\mathrm{al}^{16}\end{array}$ & $\mathrm{PC}$ & $\begin{array}{l}\text { COVID-19+ } \\
\text { adults }\end{array}$ & $\begin{array}{l}\text { Papa Giovanni XXIII } \\
\text { Hospital (Bergamo) }\end{array}$ & Italy & 767 & $\begin{array}{l}105(84- \\
127) \text { days }{ }^{c} \\
* * *\end{array}$ & $63(13.6)^{d}$ & $32.90 \%$ & $\begin{array}{l}\text { PASC and } \\
\text { symptom } \\
\text { prevalence }\end{array}$ \\
\hline & Feb 2021 & $\begin{array}{l}\text { Soraas et } \\
\mathrm{al}^{39}\end{array}$ & PC & $\begin{array}{l}\text { COVID-19+, } \\
\text { non- } \\
\text { hospitalized } \\
\text { adults }\end{array}$ & Online Survey & Norway & 588 & $\begin{array}{l}248(18) \\
\text { days }^{d * * * *}\end{array}$ & $48^{d}$ & $57 \%$ & $\begin{array}{l}\text { PASC and } \\
\text { symptom } \\
\text { prevalence }\end{array}$ \\
\hline & Mar 2021 & Morin et $\mathrm{al}^{40}$ & $\mathrm{PC}$ & $\begin{array}{l}\text { COVID-19+, } \\
\text { hospitalized } \\
\text { adults }\end{array}$ & Bicêtre Hospital (Paris) & France & 478 & $\begin{array}{l}113(94- \\
128) \text { days }{ }^{c} \\
* * *\end{array}$ & $61(16)^{d}$ & $42.10 \%$ & $\begin{array}{l}\text { PASC and } \\
\text { symptom } \\
\text { prevalence }\end{array}$ \\
\hline & Mar 2021 & Lampl et $\mathrm{al}^{41}$ & $\mathrm{RC}$ & COVID-19+ & $\begin{array}{l}\text { Regensburg Public Health } \\
\text { Department, Regensburg, } \\
\text { Bavaria }\end{array}$ & Germany & 419 & $6+$ weeks * & $44(30-57)^{c}$ & $56.60 \%$ & $\begin{array}{l}\text { PASC and } \\
\text { symptom } \\
\text { prevalence }\end{array}$ \\
\hline
\end{tabular}




\begin{tabular}{|c|c|c|c|c|c|c|c|c|c|c|c|}
\hline$\frac{5}{\circ}$ & $\begin{array}{l}\text { Date of } \\
\text { Publication }\end{array}$ & Authors & $\begin{array}{l}\text { Study } \\
\text { Design } \\
\text { a }\end{array}$ & $\begin{array}{l}\text { Population of } \\
\text { Interest }\end{array}$ & Setting & Country & Sample Size & $\begin{array}{l}\text { Follow-up } \\
\text { Time } f\end{array}$ & Age & $\begin{array}{l}\text { Sex (\% } \\
\text { female) }\end{array}$ & $\begin{array}{l}\text { Outcomes of } \\
\text { Interest } \mathrm{e}\end{array}$ \\
\hline \multirow{12}{*}{ 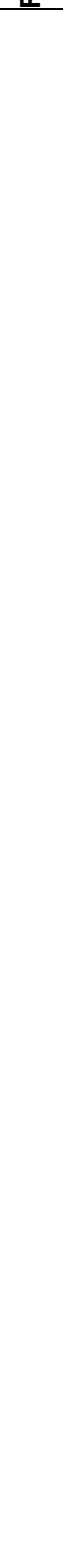 } & Mar 2021 & $\begin{array}{l}\text { Ayoubkhani } \\
\text { et al }{ }^{12}\end{array}$ & $\mathrm{RC}$ & $\begin{array}{l}\text { COVID-19+, } \\
\text { hospitalized }\end{array}$ & NHS hospitals in England & UK & 47,780 & $\begin{array}{l}140(50) \\
\text { days } d^{* * \star}\end{array}$ & $64.5(19.2)^{d}$ & $45 \%$ & $\begin{array}{l}\text { Symptom } \\
\text { prevalence }\end{array}$ \\
\hline & Apr 2021 & $\begin{array}{l}\text { Lemhofer et } \\
\mathrm{al}^{42}\end{array}$ & CS & $\begin{array}{l}\text { COVID-19+ } \\
\text { adults with mild } \\
\text { to moderate } \\
\text { infection }\end{array}$ & $\begin{array}{l}\text { Survey Administered by } 2 \\
\text { Bavarian health } \\
\text { departments }\end{array}$ & Germany & 365 & 3 months+ & $49.8(16.9)^{d}$ & $58.50 \%$ & $\begin{array}{l}\text { PASC and } \\
\text { symptom } \\
\text { prevalence }\end{array}$ \\
\hline & May 2021 & Orrù et al ${ }^{43}$ & CS & $\begin{array}{l}\text { COVID-19+ } \\
\text { adults plus } \\
\text { COVID-19- } \\
\text { controls }\end{array}$ & $\begin{array}{l}\text { Online Survey } \\
\text { (recruitment via social } \\
\text { media or email) }\end{array}$ & Italy & 507 & $\prod_{* *}^{1-3}$ months & $\begin{array}{l}0.2 \%<20 \\
12.23 \% 20-29 \\
20.91 \% 30-39 \\
30.77 \% 40-49 \\
26.04 \% 50-59 \\
8.24 \% 60-69 \\
1.58 \%>70\end{array}$ & $82.05 \%$ & $\begin{array}{l}\text { Symptom } \\
\text { prevalence }\end{array}$ \\
\hline & May 2021 & $\begin{array}{l}\text { Desgranges } \\
\text { et } \mathrm{al}^{44}\end{array}$ & $\mathrm{PC}$ & $\begin{array}{l}\text { COVID-19+, } \\
\text { symptomatic, } \\
\text { outpatient } \\
\text { adults with at } \\
\text { least one risk } \\
\text { factor for } \\
\text { severe COVID- } \\
19\end{array}$ & $\begin{array}{l}\text { University hospital of } \\
\text { Lausanne }\end{array}$ & Switzerland & 418 & $\begin{array}{l}105(121- \\
204) \text { days }{ }^{c}\end{array}$ & $41(31-54)^{c}$ & $62 \%$ & $\begin{array}{l}\text { PASC and } \\
\text { symptom } \\
\text { prevalence, risk } \\
\text { factors }\end{array}$ \\
\hline & June 2021 & $\begin{array}{l}\text { Peghin et } \\
\mathrm{al}^{45}\end{array}$ & $A C$ & $\begin{array}{l}\text { COVID-19+ } \\
\text { adults }\end{array}$ & Udine Hospital & Italy & 599 & $\begin{array}{l}191(172- \\
204) \text { days c } \\
* * \star *\end{array}$ & $53(15.8)^{d}$ & $53.40 \%$ & $\begin{array}{l}\text { PASC and } \\
\text { symptom } \\
\text { prevalence }\end{array}$ \\
\hline & June 2021 & Righi et al ${ }^{46}$ & $\mathrm{PC}$ & $\begin{array}{l}\text { COVID-19+ } \\
\text { adults }\end{array}$ & $\begin{array}{l}\text { Verona University } \\
\text { Hospital }\end{array}$ & Italy & 448 & $\begin{array}{l}6 \text { weeks, } \\
12 \text { weeks } \\
* *\end{array}$ & $56(45-66)^{c}$ & $45.10 \%$ & $\begin{array}{l}\text { PASC and } \\
\text { symptom } \\
\text { prevalence }\end{array}$ \\
\hline & June 2021 & $\begin{array}{l}\text { Maestre- } \\
\text { Muñiz et al }{ }^{47}\end{array}$ & CS & $\begin{array}{l}\text { COVID-19+ } \\
\text { adults }\end{array}$ & $\begin{array}{l}\text { Tomelloso General } \\
\text { Hospital }\end{array}$ & Spain & 543 & 12 months & $\mathrm{n} / \mathrm{a}$ & $\mathrm{n} / \mathrm{a}$ & $\begin{array}{l}\text { PASC and } \\
\text { symptom } \\
\text { prevalence }\end{array}$ \\
\hline & July 2021 & Ghosn et $\mathrm{al}^{48}$ & $\mathrm{PC}$ & $\begin{array}{l}\text { COVID-19+, } \\
\text { hospitalized }\end{array}$ & $\begin{array}{l}\text { Multicenter (French Covid } \\
\text { Cohort) }\end{array}$ & France & 1,137 & $\begin{array}{l}3 \text { months, } 6 \\
\text { months *** }\end{array}$ & $61(51-71)^{c}$ & $37 \%$ & $\begin{array}{l}\text { Symptom } \\
\text { prevalence }\end{array}$ \\
\hline & July 2021 & $\begin{array}{l}\text { Augustin et } \\
\mathrm{al}^{14}\end{array}$ & PC & $\begin{array}{l}\text { COVID-19+, } \\
\text { non- } \\
\text { hospitalized } \\
\text { adults }\end{array}$ & $\begin{array}{l}\text { University Hospital } \\
\text { Cologne }\end{array}$ & Germany & $\begin{array}{l}1.4 \text { months: } \\
958 \\
4.3 \text { months: } \\
442 \\
6.8 \text { months: } \\
353\end{array}$ & $\begin{array}{l}1.4(1-2) \\
\text { months, } \\
4.3(3-5) \\
\text { months, } \\
6.8(6-8) \\
\text { months c } \\
\star \star \star \star \star\end{array}$ & $43(31-54)^{c}$ & $53.50 \%$ & $\begin{array}{l}\text { PASC and } \\
\text { symptom } \\
\text { prevalence, risk } \\
\text { factors }\end{array}$ \\
\hline & July 2021 & $\begin{array}{l}\text { Menges et } \\
\mathrm{al}^{49}\end{array}$ & $\mathrm{PC}$ & $\begin{array}{l}\text { COVID-19+ } \\
\text { adults }\end{array}$ & $\begin{array}{l}\text { Department of Health of } \\
\text { the Canton of Zurich, } \\
\text { Switzerland Surveillance }\end{array}$ & Switzerland & 431 & $\begin{array}{l}7.2(5.9- \\
10.3) \\
\text { months c } \\
\star \star \star \star \star\end{array}$ & $47(33-58)^{c}$ & $50 \%$ & $\begin{array}{l}\text { PASC and } \\
\text { symptom } \\
\text { prevalence, risk } \\
\text { factors }\end{array}$ \\
\hline & July 2021 & Taylor et $\mathrm{al}^{50}$ & $\mathrm{PC}$ & $\begin{array}{l}\text { COVID-19+, } \\
\text { hospitalized }\end{array}$ & $\begin{array}{l}\text { Barts Health NHS Trust } \\
\text { (London) }\end{array}$ & UK & 675 & $\underset{* * \star}{12+\text { weeks }}$ & $\mathrm{n} / \mathrm{a}$ & $42.10 \%$ & $\begin{array}{l}\text { Symptom } \\
\text { prevalence }\end{array}$ \\
\hline & Aug 2021 & $\begin{array}{l}\text { Fernández- } \\
\text { de-Las- } \\
\text { Peñas et al }{ }^{51}\end{array}$ & $\mathrm{PC}$ & $\begin{array}{l}\text { COVID-19+, } \\
\text { hospitalized }\end{array}$ & Multicenter & Spain & 1,142 & $\begin{array}{l}7(0.6) \\
\text { months d } \\
\star \star \star \star \star\end{array}$ & $61(17)^{d}$ & $48 \%$ & $\begin{array}{l}\text { PASC and } \\
\text { symptom } \\
\text { prevalence }\end{array}$ \\
\hline
\end{tabular}




\begin{tabular}{|c|c|c|c|c|c|c|c|c|c|c|c|}
\hline 응 & $\begin{array}{l}\text { Date of } \\
\text { Publication }\end{array}$ & Authors & $\begin{array}{l}\text { Study } \\
\text { Design } \\
\text { a }\end{array}$ & $\begin{array}{l}\text { Population of } \\
\text { Interest }\end{array}$ & Setting & Country & Sample Size & $\begin{array}{l}\text { Follow-up } \\
\text { Time } f\end{array}$ & Age & $\begin{array}{l}\text { Sex }(\% \\
\text { female) }\end{array}$ & $\begin{array}{l}\text { Outcomes of } \\
\text { Interest } \mathrm{e}\end{array}$ \\
\hline & Apr 2021 & $\begin{array}{l}\text { Whittaker et } \\
\text { al }^{52}\end{array}$ & PC & $\begin{array}{l}\text { COVID-19+ } \\
\text { adults }\end{array}$ & $\begin{array}{l}\text { Clinical Practice } \\
\text { Research Database } \\
\text { (CPRD) Aurum }\end{array}$ & UK & 46,687 & $\begin{array}{l}63 \text { days } \\
(63-63)^{c} \text { ** }\end{array}$ & $\begin{array}{l}38.6 \% 18-30 \\
16.6 \% 31-40 \\
15.7 \% 41-50 \\
16 \% 51-60 \\
7.4 \% 61-70 \\
3.3 \% 71-80 \\
2.4 \%>80\end{array}$ & $54.60 \%$ & $\begin{array}{l}\text { Symptom } \\
\text { prevalence }\end{array}$ \\
\hline \multirow{6}{*}{ 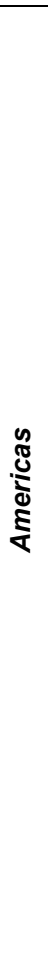 } & Dec 2020 & Cirulli et al ${ }^{53}$ & PC & $\begin{array}{l}\text { COVID-19+ } \\
\text { adults }\end{array}$ & $\begin{array}{l}\text { Surveyed participants } \\
\text { from Helix DNA Discovery } \\
\text { Project and Healthy } \\
\text { Nevada Project }\end{array}$ & USA & 357 & $\begin{array}{l}30,60,90 \\
\text { days *** }\end{array}$ & $\mathrm{n} / \mathrm{a}$ & $n / a$ & $\begin{array}{l}\text { PASC and } \\
\text { symptom } \\
\text { prevalence, risk } \\
\text { factors } \\
\end{array}$ \\
\hline & Mar 2021 & $\begin{array}{l}\text { Hirschtick et } \\
\mathrm{al}^{54}\end{array}$ & CS & $\begin{array}{l}\text { COVID-19+, } \\
\text { symptomatic } \\
\text { adults }\end{array}$ & $\begin{array}{l}\text { Michigan Disease } \\
\text { Surveillance System }\end{array}$ & USA & 593 & 30,60 days & $51.5(15.8)^{d}$ & $56.10 \%$ & $\begin{array}{l}\text { PASC } \\
\text { prevalence }\end{array}$ \\
\hline & Mar 2021 & $\begin{array}{l}\text { Spotnitz et } \\
\mathrm{al}^{15}\end{array}$ & $\mathrm{RC}$ & COVID-19+ & $\begin{array}{l}\text { ICM MarketScan } \\
\text { Commercial Claims and } \\
\text { Encounters, Optum } \\
\text { Electronic Health Record, } \\
\text { and Columbia University } \\
\text { Irving Medican Center. }\end{array}$ & USA & 448,176 & $\begin{array}{l}30-180 \\
\text { days *** }\end{array}$ & $\mathrm{n} / \mathrm{a}$ & $\mathrm{n} / \mathrm{a}$ & $\begin{array}{l}\text { PASC and } \\
\text { symptom } \\
\text { prevalence }\end{array}$ \\
\hline & Mar 2021 & Perlis et a ${ }^{20}$ & CS & $\begin{array}{l}\text { COVID-19+, } \\
\text { symptomatic }\end{array}$ & $\begin{array}{l}\text { Online Survey with Non- } \\
\text { Probability Sampling }\end{array}$ & USA & 6,211 & 10 months & $37.8(12.2)^{d}$ & $45.10 \%$ & $\begin{array}{l}\text { PASC and } \\
\text { symptom } \\
\text { prevalence, risk } \\
\text { factors } \\
\end{array}$ \\
\hline & Mar 2021 & Huang et al ${ }^{55}$ & $\mathrm{RC}$ & $\begin{array}{l}\text { COVID-19+, } \\
\text { non- } \\
\text { hospitalized } \\
\text { with 5+ year } \\
\text { history in EHR } \\
\text { system }\end{array}$ & $\begin{array}{l}\text { UC CORDS (University of } \\
\text { California Covid research } \\
\text { data set) }\end{array}$ & USA & 1,407 & $61+$ days $* *$ & $\begin{array}{l}2 \%<18 \\
10 \% 18-29 \\
16 \% 30-39 \\
18 \% 40-49 \\
21 \% 50-59 \\
16 \% 60-69 \\
12 \% 70-79 \\
6 \%>=80 \\
\end{array}$ & $58.90 \%$ & $\begin{array}{l}\text { Symptom } \\
\text { prevalence, risk } \\
\text { factors }\end{array}$ \\
\hline & Apr 2021 & $\begin{array}{l}\text { Damiano et } \\
\text { al }^{56}\end{array}$ & PC & $\begin{array}{l}\text { COVID-19+, } \\
\text { hospitalized } \\
\text { (moderate or } \\
\text { severe Covid) } \\
\text { adults }\end{array}$ & $\begin{array}{l}\text { Hospital das Clínicas da } \\
\text { Faculdade de Medicina } \\
\text { da Universidade } \\
\text { de São Paulo }\end{array}$ & Brazil & 425 & 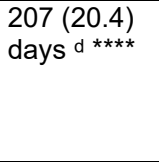 & $55.7(14.2)^{d}$ & $51.53 \%$ & $\begin{array}{l}\text { Psychiatric and } \\
\text { cognitive } \\
\text { symptom } \\
\text { prevalence }\end{array}$ \\
\hline
\end{tabular}




\begin{tabular}{|c|c|c|c|c|c|c|c|c|c|c|c|}
\hline 응 & $\begin{array}{l}\text { Date of } \\
\text { Publication }\end{array}$ & Authors & $\begin{array}{l}\text { Study } \\
\text { Design } \\
\text { a }\end{array}$ & $\begin{array}{l}\text { Population of } \\
\text { Interest }\end{array}$ & Setting & Country & Sample Size & $\begin{array}{l}\text { Follow-up } \\
\text { Time }\end{array}$ & Age & $\begin{array}{l}\text { Sex }(\% \\
\text { female) }\end{array}$ & $\begin{array}{l}\text { Outcomes of } \\
\text { Interest e }\end{array}$ \\
\hline & Apr 2021 & $\begin{array}{l}\text { Chevinsky et } \\
\mathrm{al}^{57}\end{array}$ & PC & $\begin{array}{l}\text { COVID-19+ } \\
\text { adult inpatients } \\
\text { and outpatients }\end{array}$ & $\begin{array}{l}\text { Premier Healthcare } \\
\text { Database Sepical COVID- } \\
\text { 19 Release (PHD-SR) }\end{array}$ & USA & $\begin{array}{l}\text { Outpatients: } \\
44,489 \\
\text { Inpatients: } \\
27,284\end{array}$ & $\begin{array}{l}31-60 \text { days, } \\
61-90 \text { days, } \\
91-120 \\
\text { days }{ }^{* \star \star}\end{array}$ & $\begin{array}{l}\text { Inpatients: } \\
8.8 \% 18-39 \\
10 \% 40-49 \\
28.1 \% 50-64 \\
22.1 \% 65-74 \\
18 \% 75-84 \\
13 \%>=85 \\
\\
\text { Outpatients: } \\
35.7 \% 19-39 \\
18.1 \% 40-49 \\
25.9 \% 50-64 \\
10.3 \% 65-74 \\
6 \% 75-84 \\
4 \%>=85\end{array}$ & $\begin{array}{l}52.5 \% \\
\text { (inpatients) } \\
61.2 \% \\
\text { (outpatient } \\
\text { s) }\end{array}$ & $\begin{array}{l}\text { PASC and } \\
\text { symptom } \\
\text { prevalence }\end{array}$ \\
\hline & May 2021 & $\begin{array}{l}\text { Yomogida et } \\
\mathrm{al}^{58}\end{array}$ & PC & $\begin{array}{l}\text { COVID-19+ } \\
\text { adults }\end{array}$ & $\begin{array}{l}\text { Long Beach Department } \\
\text { of Health and Human } \\
\text { Services Surveillance }\end{array}$ & USA & 366 & $\begin{array}{l}1 \text { Month, } 2 \\
\text { months, } 10 \\
\text { weeks-5 } \\
\text { months }\end{array}$ & $\begin{array}{l}11.4 \% 18-24 \\
39.3 \% 25-39 \\
30.2 \% 40-54 \\
10.6 \% 55-64 \\
8.2 \% 65+\end{array}$ & $56.40 \%$ & $\begin{array}{l}\text { PASC and } \\
\text { symptom } \\
\text { prevalence, risk } \\
\text { factors }\end{array}$ \\
\hline & June 2021 & $\begin{array}{l}\text { Wong-Chew } \\
\text { et al }\end{array}$ & PC & $\begin{array}{l}\text { COVID-19+, } \\
\text { hospitalized } \\
\text { adults }\end{array}$ & $\begin{array}{l}\text { Temporary Covid-19 } \\
\text { Hospiital in Mexico City }\end{array}$ & Mexico & $\begin{array}{l}30 \text { days: } \\
1,303 \\
90 \text { days: } \\
928\end{array}$ & 30,90 days & $\mathrm{n} / \mathrm{a}$ & $\mathrm{n} / \mathrm{a}$ & $\begin{array}{l}\text { Symptom } \\
\text { prevalence, risk } \\
\text { factors }\end{array}$ \\
\hline & June 2021 & $\begin{array}{l}\text { Shoucri et } \\
\mathrm{al}^{60}\end{array}$ & $\mathrm{RC}$ & $\begin{array}{l}\text { COVID-19+, } \\
\text { hospitalized } \\
\text { adults }\end{array}$ & $\begin{array}{l}\text { New York- } \\
\text { Presbyterian/Columbia } \\
\text { University Irving Medical } \\
\text { Center }\end{array}$ & USA & $\begin{array}{l}3 \text { months: } \\
488 \\
6 \text { months: } \\
364\end{array}$ & $\begin{array}{l}3,6 \text { months } \\
\star \star \star *\end{array}$ & $\begin{array}{l}3 \text { months: } 60 \\
(47.8-71) \\
6 \text { months: } 61 \\
(50.0-71)^{c}\end{array}$ & $\begin{array}{l}43.2 \%-3 \\
\text { months } \\
47.8 \%-6 \\
\text { months }\end{array}$ & $\begin{array}{l}\text { Symptom } \\
\text { prevalence }\end{array}$ \\
\hline \multirow[t]{2}{*}{$\stackrel{x}{\Sigma}$} & Mar 2021 & Sudre et $a 1^{18}$ & PC & $\begin{array}{l}\text { COVID-19+, } \\
\text { symptomatic }\end{array}$ & $\begin{array}{l}\text { COVID Symptom Study } \\
\text { App }\end{array}$ & $\begin{array}{l}\text { UK, Sweden, } \\
\text { US }\end{array}$ & 4,182 & $28-84$ days & $42(32-53)^{c}$ & $71.50 \%$ & $\begin{array}{l}\text { PASC and } \\
\text { symptom } \\
\text { prevalence, risk } \\
\text { factors, duration }\end{array}$ \\
\hline & May 2021 & $\begin{array}{l}\text { Taquet et } \\
\mathrm{al}^{61}\end{array}$ & $\mathrm{RC}$ & $\begin{array}{l}\text { COVID-19+, } \\
\text { age } 10+\end{array}$ & TriNetX EHR Network & USA, others & 236,379 & $\begin{array}{l}6 \text { months } \\
* * *\end{array}$ & $46(19.7)^{d}$ & $55.60 \%$ & $\begin{array}{l}\text { Risk factors, } \\
\text { duration }\end{array}$ \\
\hline
\end{tabular}

a PS = Prospective Cohort, RS = Retrospective Cohort, CS = Cross-sectional, AC = Ambidirectional Cohort

b Not all inclusion/exclusion criteria listed

c Median (IQR) or Median (range)

d Mean (SD) or Mean (95\% Cl)

e Some studies included populations and outcomes outside the scope of this review.

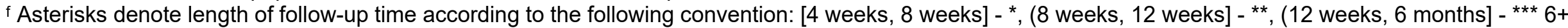
months $-{ }^{* * * *}$. If a study considered measurements at several follow-up times, the longest duration was used. 
medRxiv preprint doi: https://doi.org/10.1101/2021.11.15.21266377; this version posted November 16, 2021. The copyright holder for this preprint (which was not certified by peer review) is the author/funder, who has granted medRxiv a license to display the preprint in perpetuity.

It is made available under a CC-BY 4.0 International license.

Table 2. Meta-analysis of pooled PASC prevalence with $95 \% \mathrm{Cl}$ in COVID-19 Positive Individuals ${ }^{\text {a }}$

\begin{tabular}{|c|c|c|c|c|}
\hline & & \multicolumn{3}{|c|}{$\begin{array}{l}\text { PASC Prevalence in COVID-19 Positive Individuals [95\% Cl]; } \\
\text { (number of included studies) }\end{array}$} \\
\hline & & Any* & $\begin{array}{l}\text { Mix of Hospitalized \& } \\
\text { Non-Hospitalized }\end{array}$ & Hospitalized \\
\hline \multicolumn{2}{|l|}{ Overall } & 0.43 [0.35; 0.51]; (29) & 0.33 [0.26; 0.42]; (14) & $0.57[0.47 ; 0.66] ;(12)$ \\
\hline \multirow{2}{*}{ Sex } & Female & 0.49 [0.35; 0.63]; (9) & $\ldots$ & $\ldots$ \\
\hline & Male & 0.37 [0.24; 0.51]; (9) & $\ldots$ & $\ldots$ \\
\hline \multirow{3}{*}{ Region } & Europe & 0.43 [0.29; 0.58]; (13) & $\ldots$ & $\ldots$ \\
\hline & Asia & 0.49 [0.32; 0.66]; (7) & $\ldots$ & $\ldots$ \\
\hline & USA & 0.30 [0.21; 0.42]; (7) & $\ldots$ & $\ldots$ \\
\hline \multirow{4}{*}{ Follow-up time } & 30 days & 0.36 [0.25;0.48]; (10) & $\ldots$ & $\ldots$ \\
\hline & 60 days & 0.24 [0.13; 0.39]; (9) & $\ldots$ & $\ldots$ \\
\hline & 90 days & 0.32 [0.14; 0.57]; (9) & $\ldots$ & $\ldots$ \\
\hline & 120 days & $0.51[0.41 ; 0.61] ;(12)$ & $0.45[0.34 ; 0.57] ;(3)^{b}$ & 0.56 [0.44; 0.68]; (8) \\
\hline \multirow{6}{*}{$\begin{array}{l}\text { General } \\
\text { symptoms }\end{array}$} & Fatigue & $0.23[0.13 ; 0.38] ;(22)$ & 0.22 [0.09; 0.44]; (11) & 0.26 [0.17; 0.38]; (8) \\
\hline & Tachycardia & 0.07 [0.03; 0.18]; (6) & $\ldots$ & $\ldots$ \\
\hline & $\begin{array}{l}\text { Appetite/ } \\
\text { Eating disorder }\end{array}$ & 0.06 [0.03; 0.1]; (8) & $\cdots$ & 0.04 [0.02; 0.08]; (6) \\
\hline & Dizziness & 0.06 [0.03; 0.13]; (5) & $\ldots$ & $\ldots$ \\
\hline & Sore throat & 0.03 [0.02; 0.05]; (10) & $\ldots$ & 0.03 [0.01; 0.09]; (5) \\
\hline & Fever & 0.02 [0.01; 0.04]; (12) & 0.02 [0.01; 0.04]; (6) & $\ldots$ \\
\hline \multirow{7}{*}{$\begin{array}{l}\text { Neurologic } \\
\text { symptoms }\end{array}$} & Sleep problems & 0.13 [0.06; 0.28]; (14) & $\ldots$ & $0.16[0.11 ; 0.23] ;(9)$ \\
\hline & Memory problems & 0.13 [0.1;0.18]; (10) & $\ldots$ & 0.12 [0.09; 0.17]; (7) \\
\hline & $\begin{array}{l}\text { Concentration/ } \\
\text { Confusion / Brain } \\
\text { fog }\end{array}$ & 0.09 [0.05; 0.17]; (11) & $\ldots$ & 0.06 [0.03; 0.13]; (6) \\
\hline & Smell & 0.08 [0.05; 0.12]; (13) & 0.14 [0.06; 0.28]; (5) & 0.05 [0.03; 0.08]; (7) \\
\hline & Taste & 0.08 [0.04; 0.13$] ;(10)$ & $\ldots$ & 0.04 [0.02; 0.07]; (5) \\
\hline & Headache & 0.05 [0.03; 0.08]; (17) & 0.03 [0.01; 0.1]; (8) & 0.05 [0.02; 0.11]; (7) \\
\hline & Smell or Taste & 0.05 [0.01; 0.21]; (9) & 0.04 [0.01; 0.23]; (5) & $\ldots$ \\
\hline \multirow{3}{*}{$\begin{array}{l}\text { Respiratory } \\
\text { symptoms }\end{array}$} & Dyspnea & 0.13 [0.09; 0.19]; (24) & 0.12 [0.07; 0.2]; (13) & 0.16 [0.13; 0.21]; (8) \\
\hline & Cough & 0.07 [0.05; 0.09]; (24) & 0.06 [0.04; 0.09]; (11) & 0.07 [0.05; 0.12]; (10) \\
\hline & Chest pain & 0.05 [0.04; 0.07]; (15) & 0.03 [0.02; 0.07]; (6) & 0.07 [0.05; 0.1]; (9) \\
\hline \multirow{2}{*}{$\begin{array}{l}\text { Psychological } \\
\text { symptoms }\end{array}$} & Anxiety & 0.10 [0.06; 0.16]; (10) & 0.12 [0.05; 0.25]; (6) & $\ldots$ \\
\hline & Depression & 0.10 [0.05; 0.21]; (7) & $0.13[0.04 ; 0.34] ;(5)$ & $\ldots$ \\
\hline \multirow{2}{*}{$\begin{array}{l}\text { Musculoskeletal } \\
\text { symptoms }\end{array}$} & Joint pain & 0.13 [0.05; 0.29]; (5) & $\ldots$ & $\ldots$ \\
\hline & Myalgia & 0.06 [0.03; 0.13]; (17) & 0.06 [0.02; 0.16]; (10) & 0.07 [0.04; 0.12]; (7) \\
\hline \multirow{2}{*}{$\begin{array}{l}\text { Gastrointestinal } \\
\text { symptoms }\end{array}$} & Abdominal pain & 0.04 [0.02; 0.12]; (6) & $\ldots$ & $\ldots$ \\
\hline & Diarrhea & 0.03 [0.01; 0.07]; (9) & $\ldots$ & $0.02[0.01 ; 0.04] ;(5)$ \\
\hline $\begin{array}{l}\text { Dermatologic } \\
\text { symptoms }\end{array}$ & Hair loss & 0.07 [0.02; 0.24]; (10) & $\ldots$ & $0.13[0.08 ; 0.21] ;(6)$ \\
\hline
\end{tabular}

* Includes studies that reported on only non-hospitalized, mix of hospitalized \& non-hospitalized, or only hospitalized COVID-19 positive patients

a Pooled estimates and 95\% Cls calculated from random-effect models with inverse variance weighting as described in methods. Prevalence is stratified by acute-phase hospitalization status. Estimates for the non-hospitalized population are not provided due to lack of sample size.

${ }^{b}$ Only 3 studies with mixed hospitalized and non-hospitalized population. This estimate should be interpreted with caution due to low sample size. 


$\begin{array}{ll}\text { Studies } & \text { Prevalence }(95 \% \\ \text { Fernández-de-Las-Peñas et al Spain } & 0.81[0.79 ; 0.84] \\ \text { Huang et al China } & 0.76[0.74 ; 0.78] \\ \text { Wong-Chew et al Mexico } & 0.76[0.74 ; 0.78] \\ \text { Ghosn et al France } & 0.68[0.65 ; 0.71] \\ \text { Areekal et al India } & 0.66[0.61 ; 0.71] \\ \text { Lemhofer et al Germany } & 0.62[0.57 ; 0.67] \\ \text { Munblit et al Russia } & 0.58[0.56 ; 0.60] \\ \text { Maestre-Muñiz et al Spain } & 0.57[0.53 ; 0.61] \\ \text { Zheng et al China } & 0.56[0.52 ; 0.60] \\ \text { Desgranges et al Switzerland } & 0.53[0.48 ; 0.58] \\ \text { Hirschtick et al USA } & 0.52[0.48 ; 0.57] \\ \text { Venturelli et al Italy } & 0.51[0.48 ; 0.55] \\ \text { Morin et al France } & 0.51[0.46 ; 0.56] \\ \text { Xiong et al China } & 0.50[0.45 ; 0.54] \\ \text { Yomogida et al USA } & 0.48[0.43 ; 0.53] \\ \text { Budhiraja et al India } & 0.40[0.37 ; 0.43] \\ \text { Peghin et al Europe } & 0.40[0.36 ; 0.44] \\ \text { Righi et al Europe } & 0.39[0.35 ; 0.44] \\ \text { Menges et al Switzerland } & 0.39[0.34 ; 0.43] \\ \text { Cirulli et al USA } & 0.36[0.31 ; 0.41] \\ \text { Shang et al China } & 0.33[0.30 ; 0.36] \\ \text { Augustin et al Europe } & 0.28[0.24 ; 0.32] \\ \text { Spotnitz et al USA } & 0.28[0.27 ; 0.28] \\ \text { Huang et al California } & 0.27[0.25 ; 0.30] \\ \text { Shoucri et al USA } & 0.25[0.22 ; 0.30] \\ \text { Naik et al } \square \text { India } & 0.22[0.20 ; 0.24] \\ \text { Sudre et al UK/SE/US } & 0.13[0.12 ; 0.14] \\ \text { Perlis et al USA } & 0.09[0.08 ; 0.10] \\ \text { Lampl et al Germany } & 0.09[0.06 ; 0.12] \\ \text { Total } & 0.43[0.35 ; 0.51] \\ \text { Heterogeneity: } \chi_{28}^{2}=8129.75(P<.001), I^{2}=100 \% \\ \end{array}$

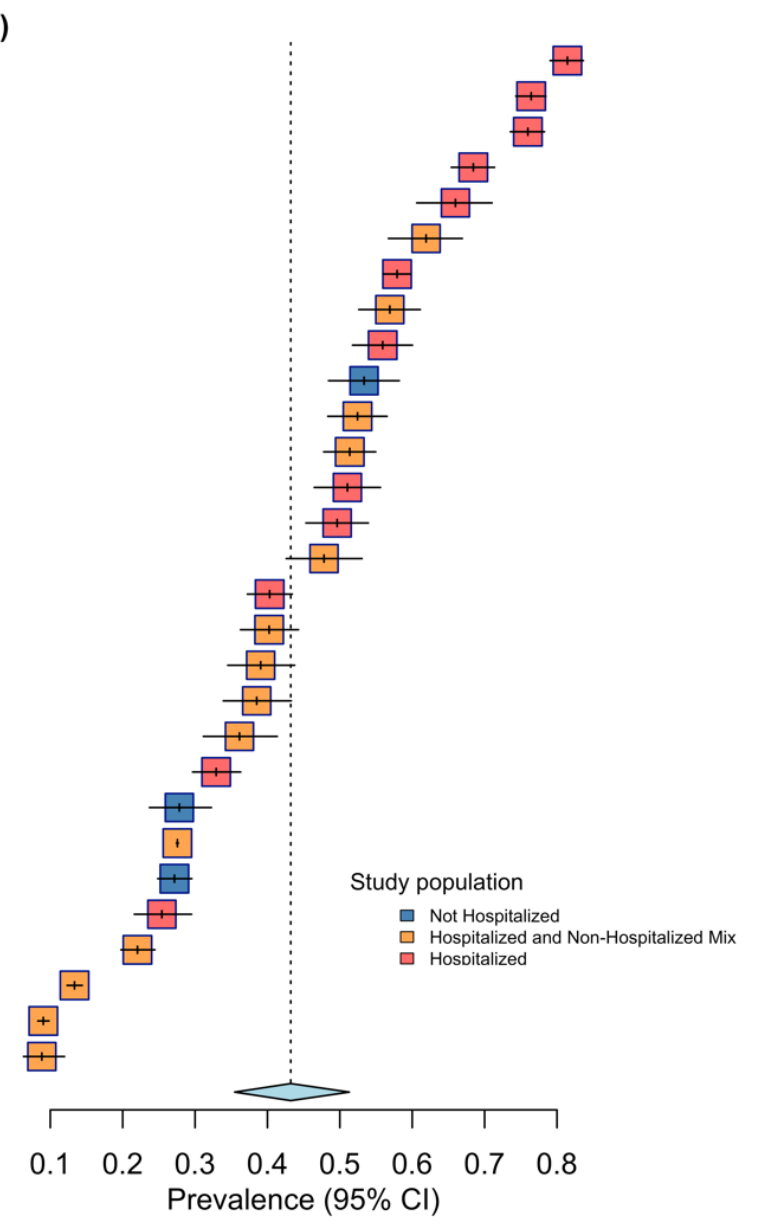

Figure 2. Forest plot for worldwide PASC prevalence.

Notes: Prevalence estimates and $95 \% \mathrm{Cls}$ are provided for each study with a relevant measure, and for the meta-analysis of all such studies. For individual studies, the horizontal line represents the estimate, whiskers represent the confidence interval, the size of the box represents the weight assigned to the study, and the color shading reflects the hospitalization status of the study population, as noted in the legend. For the pooled estimate, the width of the diamond represents the confidence interval. Meta-analyzed prevalence and $95 \% \mathrm{Cls}$ are calculated using random-effects models with inverse variance weighting as described in the methods. Measures of heterogeneity of prevalence estimates are provided. 
medRxiv preprint doi: https://doi.org/10.1101/2021.11.15.21266377; this version posted November 16, 2021. The copyright holder for this preprint (which was not certified by peer review) is the author/funder, who has granted medRxiv a license to display the preprint in perpetuity.

It is made available under a CC-BY 4.0 International license .

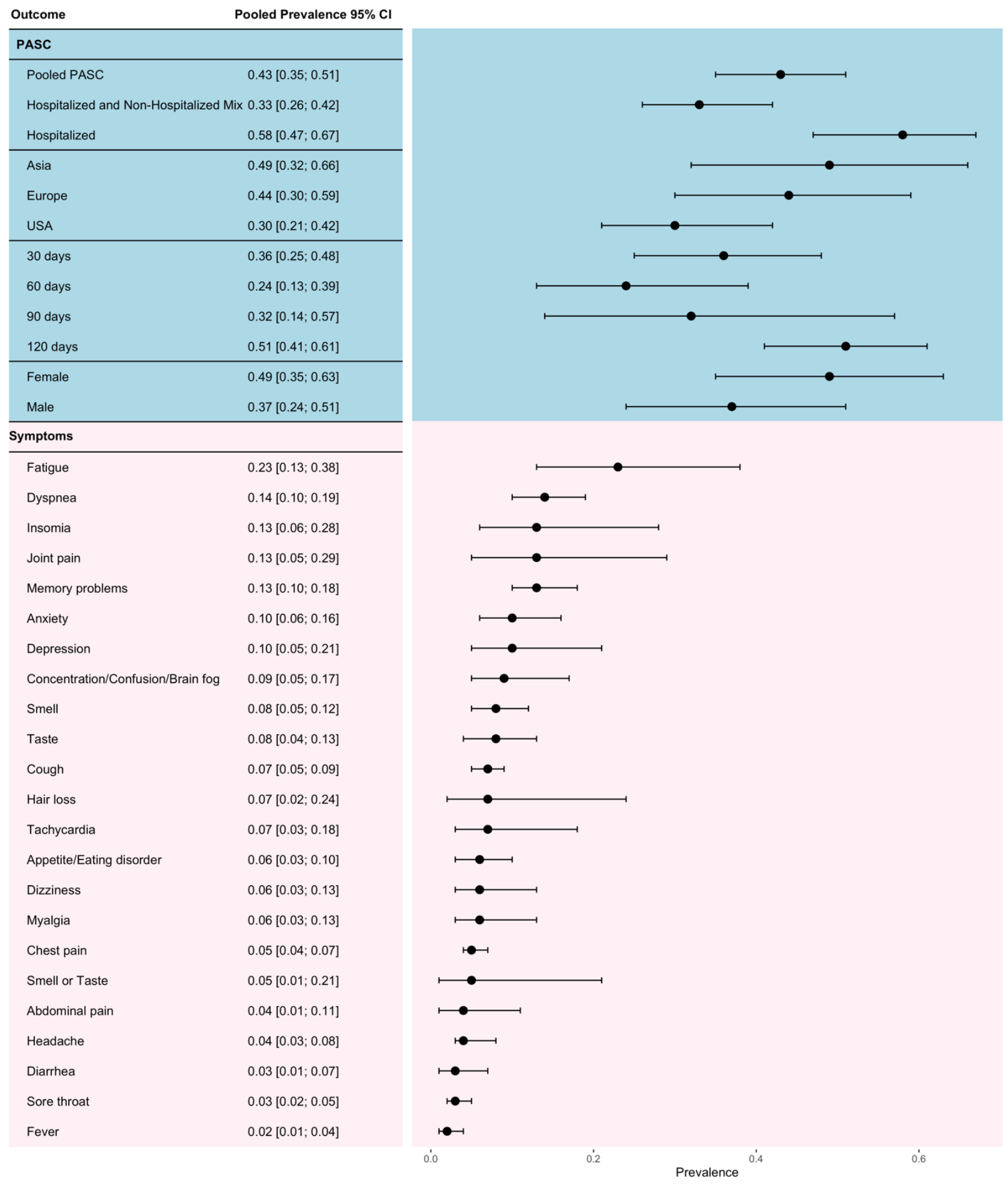

Figure 3. Forest plot for PASC prevalence by hospitalization status, region, follow-up time, and sex, as well as symptom-specific prevalence.

Notes: Pooled estimates and $95 \%$ Cls calculated from random-effect models with inverse variance weighting as described in methods. Pooled estimates with confidence intervals are provided on the left, and visualization of the intervals on the right. 


\section{References}

1. CDC. Coronavirus Disease 2019 (COVID-19) - Symptoms. Centers for Disease Control and Prevention. Published February 22, 2021. Accessed October 20, 2021. https://www.cdc.gov/coronavirus/2019-ncov/symptoms-testing/symptoms.html

2. Ramos-Casals $M$, Brito-Zerón $P$, Mariette $X$. Systemic and organ-specific immune-related manifestations of COVID-19. Nat Rev Rheumatol. 2021;17(6):315-332. doi:10.1038/s41584-021-00608-z

3. WHO Coronavirus (COVID-19) Dashboard. Accessed October 20, 2021. https://covid19.who.int

4. CDC. Healthcare Workers. Centers for Disease Control and Prevention. Published February 11, 2020. Accessed October 20, 2021. https://www.cdc.gov/coronavirus/2019ncov/hcp/clinical-care/post-covid-conditions.html

5. planning-scenarios-2020-05-20.pdf. Accessed November 8, 2021.

https://www.cdc.gov/coronavirus/2019-ncov/hcp/planning-scenarios-archive/planningscenarios-2020-05-20.pdf

6. McElfish PA, Purvis R, James LP, Willis DE, Andersen JA. Perceived Barriers to COVID-19 Testing. Int J Environ Res Public Health. 2021;18(5):2278. doi:10.3390/ijerph18052278

7. Lieberman-Cribbin W, Tuminello S, Flores RM, Taioli E. Disparities in COVID-19 Testing and Positivity in New York City. Am J Prev Med. 2020;59(3):326-332.

doi:10.1016/j.amepre.2020.06.005

8. SARS-CoV-2 infection fatality rates in India: systematic review, meta-analysis and modelbased estimation | medRxiv. Accessed November 8, 2021. https://www.medrxiv.org/content/10.1101/2021.09.08.21263296v1

9. Rahmandad H, Lim TY, Sterman J. Behavioral Dynamics of COVID-19: Estimating UnderReporting, Multiple Waves, and Adherence Fatigue Across 92 Nations. Social Science Research Network; 2021. doi:10.2139/ssrn.3635047

10. Asymptomatic SARS-CoV-2 infection: A systematic review and meta-analysis | PNAS. Accessed November 8, 2021. https://www.pnas.org/content/118/34/e2109229118

11. Carfi A, Bernabei R, Landi F, for the Gemelli Against COVID-19 Post-Acute Care Study Group. Persistent Symptoms in Patients After Acute COVID-19. JAMA. 2020;324(6):603605. doi:10.1001/jama.2020.12603

12. Ayoubkhani D, Khunti K, Nafilyan V, et al. Post-covid syndrome in individuals admitted to hospital with covid-19: retrospective cohort study. BMJ. 2021;372:n693-n693.

13. López-León S, Wegman-Ostrosky T, Perelman C, et al. More than 50 Long-Term Effects of COVID-19: A Systematic Review and Meta-Analysis. Social Science Research Network; 2021. doi:10.2139/ssrn.3769978 
medRxiv preprint doi: https://doi.org/10.1101/2021.11.15.21266377; this version posted November 16, 2021. The copyright holder for this preprint (which was not certified by peer review) is the author/funder, who has granted medRxiv a license to display the preprint in perpetuity.

It is made available under a CC-BY 4.0 International license.

14. Augustin M, Schommers P, Stecher M, et al. Post-COVID syndrome in non-hospitalised patients with COVID-19: a longitudinal prospective cohort study. Lancet Reg Health Eur. 2021;6:100122-100122.

15. Spotnitz ME, Hripcsak G, Ryan PB, Natarajan K. Characterizing Post-Acute Sequelae of SARS-CoV-2 Infection across Claims and Electronic Health Record Databases. Published online March 2021.

16. Venturelli S, Benatti SV, Casati M, et al. Surviving COVID-19 in Bergamo province: a postacute outpatient re-evaluation. Epidemiol Infect. 2021;149:e32-e32.

17. Huang $C$, Huang L, Wang $Y$, et al. 6-month consequences of COVID-19 in patients discharged from hospital: a cohort study. Lancet Lond Engl. 2021;397(10270):220-232.

18. Sudre $\mathrm{CH}$, Murray B, Varsavsky T, et al. Attributes and predictors of long COVID. Nat Med. 2021;27(4):626-631.

19. Nasserie T, Hittle M, Goodman SN. Assessment of the Frequency and Variety of Persistent Symptoms Among Patients With COVID-19: A Systematic Review. JAMA Netw Open. 2021;4(5):e2111417. doi:10.1001/jamanetworkopen.2021.11417

20. Perlis RH, Green J, Santillana M, et al. Persistence of symptoms up to 10 months following acute COVID-19 illness. MedRxiv Prepr Serv Health Sci. Published online March 2021.

21. BERGER Z, ALTIERY DE JESUS V, ASSOUMOU SA, GREENHALGH T. Long COVID and Health Inequities: The Role of Primary Care. Milbank Q. 2021;99(2):519-541.

doi:10.1111/1468-0009.12505

22. Page MJ, McKenzie JE, Bossuyt PM, et al. The PRISMA 2020 statement: an updated guideline for reporting systematic reviews. Syst Rev. 2021;10(1):89. doi:10.1186/s13643021-01626-4

23. Library Resources. Accessed October 20, 2021.

https://covid.yale.edu/research/resources/library-resources/

24. Ouzzani M, Hammady H, Fedorowicz Z, Elmagarmid A. Rayyan-a web and mobile app for systematic reviews. Syst Rev. 2016;5(1). doi:10.1186/s13643-016-0384-4

25. Schwarzer G. Meta: General Package for Meta-Analysis.; 2021. Accessed October 20, 2021. https://CRAN.R-project.org/package=meta

26. Wang N. How to Conduct a Meta-Analysis of Proportions in R: A Comprehensive Tutorial.; 2018. doi:10.13140/RG.2.2.27199.00161

27. Viechtbauer W. Metafor: Meta-Analysis Package for R.; 2021. Accessed October 20, 2021. https://CRAN.R-project.org/package=metafor

28. Martin J. (C) Joanna Briggs Institute 2017

Critical

Appraisal Checklist for Prevalence Studies. Published online 2017:7. 
medRxiv preprint doi: https://doi.org/10.1101/2021.11.15.21266377; this version posted November 16, 2021. The copyright holder for this preprint (which was not certified by peer review) is the author/funder, who has granted medRxiv a license to display the preprint in perpetuity.

It is made available under a CC-BY 4.0 International license.

29. Huang D, Chen C, Xuan W, et al. Long-Term Outcomes and Sequelae for 464 COVID-19 Patients Discharged from Leishenshan Hospital in Wuhan, China. Published online December 2020.

30. Xiong Q, Xu M, Li J, et al. Clinical sequelae of COVID-19 survivors in Wuhan, China: a single-centre longitudinal study. Clin Microbiol Infect Off Publ Eur Soc Clin Microbiol Infect Dis. 2021;27(1):89-95.

31. Zheng D, Zhang L, Feng B, et al. Long-Term Clinical Sequelae and Immunological Features of COVID-19 Survivors: A Cross-Sectional Study in Wuhan, China. Published online January 2021.

32. Shang YF, Liu T, Yu JN, et al. Half-year follow-up of patients recovering from severe COVID-19: Analysis of symptoms and their risk factors. J Intern Med. Published online April 2021.

33. Areekal B, Sukumaran ST, Andrews AM, et al. Persistence of symptoms after acute COVID19 infection- An experience from a tertiary care centre in South India. $J$ Clin Diagn Res. 2021;15(6):LC05-LC08.

34. Budhiraja S, Aggarwal M, Wig R, et al. Long Term Health Consequences of COVID-19 in Hospitalized Patients from North India: A follow up study of upto 12 months. Published online June 2021.

35. Naik S, Soneja M, Haldar S, et al. Post COVID-19 sequelae: A prospective observational study from Northern India. Published online July 2021.

36. Mannan A, M Hamidullah Mehedi $\mathrm{H}$, Chy NUHA, et al. A multi-centric, cross-sectional study on COVID-19 in Bangladesh: Clinical epidemiology and short-term outcomes in recovered individuals. Published online October 2020.

37. Sami R, Soltaninejad F, Amra B, et al. A one-year hospital-based prospective COVID-19 open-cohort in the Eastern Mediterranean region: The Khorshid COVID Cohort (KCC) study. PloS One. 2020;15(11):e0241537-e0241537.

38. Munblit D, Bobkova P, Spiridonova E, et al. Incidence and risk factors for persistent symptoms in adults previously hospitalized for COVID-19. Clin Exp Allergy. 2021;51(9):1107-1120. doi:10.1111/cea.13997

39. Soraas A, Bo R, Kalleberg KT, Ellingjord Dale M, Landro NI. Self-reported Memory Problems Eight Months after Non-Hospitalized COVID-19 in a Large Cohort. Published online February 2021.

40. Morin L, Savale L, Pham T, et al. Four-Month Clinical Status of a Cohort of Patients After Hospitalization for COVID-19. JAMA. 2021;325(15):1525-1534.

41. Lampl BMJ, Buczovsky M, Martin G, Schmied H, Leitzmann M, Salzberger B. Clinical and epidemiological data of COVID-19 from Regensburg, Germany: a retrospective analysis of 1084 consecutive cases. Infection. Published online March 2021:1-9. 
42. Lemhofer C, Sturm C, Loudovici Krug D, Best N, Gutenbrunner C. The Impact of LongCOVID on Functioning - Results from a Community Survey in Patients After Mild and Moderate SARS-CoV-2-Infection in Germany. Published online April 2021.

43. Orrù G, Bertelloni D, Diolaiuti F, et al. Long-COVID Syndrome? A Study on the Persistence of Neurological, Psychological and Physiological Symptoms. Healthc Basel Switz. $2021 ; 9(5)$.

44. Desgranges F, Tadini E, Munting A, et al. Post-COVID-19 syndrome in outpatients: a cohort study. Published online April 2021.

45. Peghin M, Palese A, Venturini M, et al. Post-COVID-19 symptoms 6 months after acute infection among hospitalized and non-hospitalized patients. Clin Microbiol Infect Off Publ Eur Soc Clin Microbiol Infect Dis. Published online June 2021.

46. Righi E, Mirandola M, Mazzaferri F, et al. Long-Term Patient-Centred Follow-up in a Prospective Cohort of Patients with COVID-19. Infect Dis Ther. Published online June 2021:1-12.

47. Maestre-Muñiz MM, Arias Á, Mata-Vázquez E, et al. Long-Term Outcomes of Patients with Coronavirus Disease 2019 at One Year after Hospital Discharge. J Clin Med. 2021;10(13).

48. Ghosn J, Piroth L, Epaulard O, et al. Persistent COVID-19 symptoms are highly prevalent 6 months after hospitalization: results from a large prospective cohort. Clin Microbiol Infect Off Publ Eur Soc Clin Microbiol Infect Dis. Published online April 2021.

49. Menges D, Ballouz T, Anagnostopoulos A, et al. Burden of Post-COVID-19 Syndrome and Implications for Healthcare Service Planning: A Population-based Cohort Study. Published online March 2021.

50. Taylor RR, Trivedi B, Patel N, et al. Post-COVID symptoms reported at asynchronous virtual review and stratified follow-up after COVID-19 pneumonia. Clin Med Lond Engl. Published online June 2021.

51. Fernández-de-Las-Peñas C, Palacios-Ceña D, Gómez-Mayordomo V, et al. Long-term postCOVID symptoms and associated risk factors in previously hospitalized patients: A multicenter study. J Infect. Published online May 2021.

52. Whittaker HR, Gulea C, Koteci A, et al. Post-Acute COVID-19 Sequelae in Cases Managed in the Community or Hospital in the UK: A Population Based Study. Published online April 2021.

53. Cirulli ET, Barrett KMS, Riffle S, et al. Long-term COVID-19 symptoms in a large unselected population. Published online October 2020.

54. Hirschtick JL, Titus AR, Slocum E, et al. Population-based estimates of post-acute sequelae of SARS-CoV-2 infection (PASC) prevalence and characteristics: A cross-sectional study. Published online March 2021. 
55. Huang Y, Pinto MD, Borelli JL, et al. COVID Symptoms, Symptom Clusters, and Predictors for Becoming a Long-Hauler: Looking for Clarity in the Haze of the Pandemic. MedRxiv Prepr Serv Health Sci. Published online March 2021.

56. Damiano RF, Caruso MJG, Cincoto AV, et al. Psychiatric and Cognitive Morbidity Following Moderate and Severe SARS-CoV-2 Infection: A Cohort Study. Published online April 2021.

57. Chevinsky JR, Tao G, Lavery AM, et al. Late conditions diagnosed 1-4 months following an initial COVID-19 encounter: a matched cohort study using inpatient and outpatient administrative data - United States, March 1-June 30, 2020. Clin Infect Dis Off Publ Infect Dis Soc Am. Published online April 2021.

58. Yomogida KS, Zhu S, Rubino F, Figueroa W, Barin N, Holman E. Longitudinal Surveillance of Post-Acute Sequelae of SARS-CoV-2 (PASC) Among Long Beach City Residents, April 1, 2020-December 10, 2020. Published online May 2021.

59. Wong-Chew RM, Cabrera EXR, Valdez CAR, et al. Symptom Cluster Analysis of long COVID-19 in Patients Discharged from the Temporary COVID-19 Hospital in Mexico City: A Longitudinal Study. Published online June 2021.

60. Shoucri SM, Purpura L, DeLaurentis C, et al. Characterising the long-term clinical outcomes of 1190 hospitalised patients with COVID-19 in New York City: a retrospective case series. BMJ Open. 2021;11(6):e049488-e049488.

61. Taquet M, Geddes JR, Husain M, Luciano S, Harrison PJ. 6-month neurological and psychiatric outcomes in 236379 survivors of COVID-19: a retrospective cohort study using electronic health records. Lancet Psychiatry. 2021;8(5):416-427.

62. Higgins JPT, Thompson SG, Deeks JJ, Altman DG. Measuring inconsistency in metaanalyses. BMJ. 2003;327(7414):557-560. doi:10.1136/bmj.327.7414.557

63. López-León S, Wegman-Ostrosky T, Perelman C, et al. More than 50 Long-Term Effects of COVID-19: A Systematic Review and Meta-Analysis. Social Science Research Network; 2021. doi:10.2139/ssrn.3769978

64. Iqbal FM, Lam K, Sounderajah V, Clarke JM, Ashrafian H, Darzi A. Characteristics and predictors of acute and chronic post-COVID syndrome: A systematic review and metaanalysis. EClinicalMedicine. 2021;36:100899. doi:10.1016/j.eclinm.2021.100899

65. Taquet M, Dercon Q, Luciano S, Geddes JR, Husain M, Harrison PJ. Incidence, cooccurrence, and evolution of long-COVID features: A 6-month retrospective cohort study of 273,618 survivors of COVID-19. PLOS Med. 2021;18(9):e1003773. doi:10.1371/journal.pmed.1003773

66. Huang L, Yao Q, Gu X, et al. 1-year outcomes in hospital survivors with COVID-19: a longitudinal cohort study. The Lancet. 2021;398(10302):747-758. doi:10.1016/S01406736(21)01755-4

67. Nalbandian A, Sehgal K, Gupta A, et al. Post-acute COVID-19 syndrome. Nat Med. 2021;27(4):601-615. doi:10.1038/s41591-021-01283-z 
medRxiv preprint doi: https://doi.org/10.1101/2021.11.15.21266377; this version posted November 16, 2021. The copyright holder for this preprint (which was not certified by peer review) is the author/funder, who has granted medRxiv a license to display the preprint in perpetuity.

It is made available under a CC-BY 4.0 International license .

68. Antonelli M, Penfold RS, Merino J, et al. Risk factors and disease profile of post-vaccination SARS-CoV-2 infection in UK users of the COVID Symptom Study app: a prospective, community-based, nested, case-control study. Lancet Infect Dis. 2021;0(0).

doi:10.1016/S1473-3099(21)00460-6

69. Six-month sequelae of post-vaccination SARS-CoV-2 infection: a retrospective cohort study of 10,024 breakthrough infections | medRxiv. Accessed November 4, 2021. https://www.medrxiv.org/content/10.1101/2021.10.26.21265508v1.full

70. Joober R, Schmitz N, Annable L, Boksa P. Publication bias: What are the challenges and can they be overcome? J Psychiatry Neurosci JPN. 2012;37(3):149-152.

doi:10.1503/jpn.120065

71. Lund LC, Hallas J, Nielsen $\mathrm{H}$, et al. Post-acute effects of SARS-CoV-2 infection in individuals not requiring hospital admission: a Danish population-based cohort study. Lancet Infect Dis. 2021;21(10):1373-1382. doi:10.1016/S1473-3099(21)00211-5

72. Suhail Y, Afzal J, Kshitiz. Incorporating and addressing testing bias within estimates of epidemic dynamics for SARS-CoV-2. BMC Med Res Methodol. 2021;21(1):11. doi:10.1186/s12874-020-01196-4 
medRxiv preprint doi: https://doi.org/10.1101/2021.11.15.21266377; this version posted November 16, 2021. The copyright holder for this preprint (which was not certified by peer review) is the author/funder, who has granted medRxiv a license to display the preprint in perpetuity.

It is made available under a CC-BY 4.0 International license .

\section{Acknowledgements}

The authors thank the librarians from the University of Michigan Taubman Health Sciences Library, and in particular, Judith E. Smith, for their guidance on constructing the search strategy for this systematic review.

\section{Author Contributions}

Conceptualization: XS, LGF, BM

Methodology: XS, LGF, BM

Investigation: CC (Screener 1), SRH (Screener 2), XS, LGF, BM

Supervision: XS, LGF, BM

Writing - original draft: CC, SRH, LZ, XS, LGF, BM

Writing - review \& editing: CC, SRH, LZ, XS, LGF, BM

\section{Funding/Support}

The research was sponsored by funding from the University of Michigan School of Public Health and Center for Precision Health Data Science.

\section{Conflict of Interest Disclosures}

Authors have no competing interests 


\section{Supplementary Online Content}

Prevalence and Risk Factors for Post-Acute Sequela of COVID-19 (PASC): A Systematic Review and Meta-Analysis

eMethods 1. Systematic review procedure

eMethods 2. Sample size calculation

eMethods 3. Meta-analysis framework

eMethods 4. Risk of bias assessment across included articles

eTable 1. PRISMA checklist

eTable 2. Characterization and synonyms of symptoms

eTable 3. Summary of excluded articles by region and follow-up time

eTable 4. Summary of significant non-meta-analyzed risk factors for PASC

eTable 5. Summary of duration of PASC and symptoms

eFigure 1. Stratified analysis of PASC by persisted versus not persisted symptoms

eFigure 2. Funnel plot for publication bias assessment among included studies

eFigure 3. PRISMA flow diagram for extended search

eFigure 4. Forest plots with the prevalence estimates of long COVID-19 stratified by (A) sex, (B) hospitalization, (C) region, and (D) follow-up time, respectively

eFigure 5. Meta-analysis of studies with 120 follow-up days stratified by acute-phase hospitalization status of study population

eFigure 6. Supplementary meta-analysis of PASC symptom-specific prevalence eFigure 7. Forest plots of risk factors for PASC by a) female and b) asthma eReferences

This supplemental material has been provided by the authors to give readers additional information about their work. 


\section{eMethods 1. Systematic review procedure}

To perform the systematic review presented herein, we collected both publications and preprints systematically that concern prevalence, risk factors, and/or duration of PASC in any country worldwide. In identifying relevant articles, we searched the following three databases: PubMed, Embase, and iSearch for preprints (encompassing bioRxiv, medRxiv, preprints.org, Research Square, SSRN). The search was conducted on July 5,2021 . Hence, the resulting captured studies reflect those available from January 1 , 2020 to July 5, 2021. A second search was conducted on August 12, 2021, to ensure we would not fail to capture recent, important studies. In this search, we used the same search terms and filters as our July 5 search and restricted our attention to studies published in well-established medical journals (i.e., JAMA, Lancet, NEJM, Nature, BMJ, and PloS). Details of study inclusion from the August 12 search can be found in eFigure 3. Upon securing citations from the search engines into Mendeley ${ }^{1}$, the reference manager, the resulting body of citations were deduplicated, and subsequently imported into the online tool Rayyan ${ }^{2}$ for screening. Search blocks and filters for PubMed, Embase, and iSearch are detailed below.

PubMed

Date searched: $7 / 5 / 2021$

Number of results: 2,884

Date filter: January 1, 2020 to present

Other filters applied: Language $=$ English

\section{Search blocks}

1. covid-19[tw] OR COVID19[tw] OR SARS-CoV-2[tw] OR SARS-CoV2[tw] OR severe acute respiratory syndrome coronavirus 2[tw] OR 2019-nCoV[tw] OR 2019nCoV[tw] OR coronavirus[tw] OR coronavirus[mh] OR covid-19[mh] OR covid[tw]

2. “long COVID"[tw] OR "long covid-19"[tw] OR "long-covid"[tw] OR "long-covid-19"[tw] OR "long haul" [tw] OR “long hauler"[tw] OR "long haulers"[tw] OR "long-haul”[tw] OR "long-hauler" [tw] OR “long-haulers"[tw] OR "chronic COVID"[tw] OR "chronic covid-19"[tw] OR "post-acute COVID"[tw] OR "post-acute covid-19"[tw] OR "post acute COVID"[tw] OR "post acute covid-19"[tw] OR "persistent COVID"[tw] OR "persistent covid-19"[tw] OR "post-COVID"[tw] OR "post-covid-19"[tw] OR "post COVID"[tw] OR "post covid-19"[tw] OR "sequela"[tw] OR "sequelae"[tw] OR "longterm"[tw] OR "long term" [tw] OR "covid syndrome"[tw] OR "covid-19 syndrome"[tw] OR "postacute COVID-19 syndrome" [Supplementary Concept] OR "persistent symptom"[tw] OR "persistent symptoms"[tw] OR "PASC"[tw] OR "PACS"[tw] OR "PPCS"[tw] OR "post-acute"[tw] OR "post acute"[tw]

3. "prevalent"[tw] OR "prevalence"[tw] OR "prevalence"[mh] OR "occurrence"[tw] OR "occurrences"[tw] OR "duration"[tw] OR "durations"[tw] OR "length"[tw] OR "lengths"[tw] OR "risk factor" [tw] OR "risk factors"[tw] OR ("risk"[tw] AND "factor"[tw]) OR ("risk"[tw] AND "factors"[tw])OR "Risk Factors"[mh] OR "predict"[tw] OR "prediction"[tw] OR "predictions"[tw] OR "predicting"[tw] OR "predictive"[tw] OR "predictor"[tw] OR "predictors"[tw] OR "symptom"[tw] OR "symptoms" [tw] OR "define"[tw] OR "defining"[tw] OR "definition"[tw] OR "definitions" [tw] OR "follow up"[tw] OR "follow-up"[tw] OR "followed up"[tw] 


\section{Embase}

Date searched: $7 / 5 / 2021$

Number of results: 1,390

Date filter: January 1, 2020 to present

Other filters applied: Language $=$ English, Embase ONLY

1. 'covid 19':ti,ab, de,tn OR covid19:ti,ab, de,tn OR 'sars cov 2':ti,ab, de,tn OR 'sars cov2':ti,ab, de,tn OR 'severe acute respiratory syndrome coronavirus 2':ti,ab, de,tn OR '2019 ncov':ti,ab, de,tn OR 2019ncov:ti,ab,de,tn OR coronavirus:ti,ab,de,tn OR 'coronavirinae'/exp OR 'coronavirus disease 2019'/exp OR covid:ti,ab,de,tn

2. 'Iong covid-19':ti,ab, de,tn OR 'long covid':ti,ab, de,tn OR 'long covid 19':ti,ab, de,tn OR 'long haul':ti,ab, de,tn OR 'long hauler':ti,ab, de,tn OR 'long haulers':ti,ab,de,tn OR 'chronic covid':ti,ab, de,tn OR 'chronic covid-19':ti,ab, de,tn OR 'post-acute covid':ti,ab, de,tn OR 'postacute covid-19':ti,ab, de,tn OR 'post acute covid':ti,ab, de,tn OR 'post acute covid19':ti,ab, de,tn OR 'persistent covid':ti,ab, de,tn OR 'persistent covid-19':ti,ab, de,tn OR 'post covid 19':ti,ab, de,tn OR 'post covid':ti,ab, de,tn OR 'post covid-19':ti,ab, de,tn OR sequela:ti,ab,de,tn OR sequelae:ti,ab,de,tn OR 'long term':ti,ab,de,tn OR 'covid syndrome':ti,ab, de,tn OR 'covid-19 syndrome':ti,ab,de,tn OR 'persistent symptom':ti,ab,de,tn OR 'persistent symptoms':ti,ab,de,tn OR pasc:ti,ab,de,tn OR pacs:ti,ab,de,tn OR ppcs:ti,ab,de,tn OR 'post acute':ti,ab,de,tn

3. prevalent:ti,ab,de,tn OR prevalence:ti,ab,de,tn OR 'prevalence'/exp OR occurrence:ti,ab,de,tn OR occurrences:ti,ab, de,tn OR duration:ti,ab,de,tn OR durations:ti,ab, de,tn OR length:ti,ab,de,tn OR lengths:ti,ab,de,tn OR 'risk factor':ti,ab, de,tn OR 'risk factors':ti,ab, de,tn OR (risk:ti,ab,de,tn AND factor:ti,ab, de,tn) OR (risk:ti,ab,de,tn AND factors:ti,ab,de,tn) OR 'risk factor'/exp OR predict:ti,ab,de,tn OR prediction:ti,ab,de,tn OR predictions:ti,ab,de,tn OR predicting:ti,ab,de,tn OR predictive:ti,ab,de,tn OR predictor:ti,ab,de,tn OR predictors:ti,ab,de,tn OR symptom:ti,ab,de,tn OR symptoms:ti,ab,de,tn OR define:ti,ab,de,tn OR defining:ti,ab,de,tn OR definition:ti,ab,de,tn OR definitions:ti,ab,de,tn OR 'follow up':ti,ab, de,tn OR 'followed up':ti,ab,de,tn 
medRxiv preprint doi: https://doi.org/10.1101/2021.11.15.21266377; this version posted November 16, 2021. The copyright holder for this preprint (which was not certified by peer review) is the author/funder, who has granted medRxiv a license to display the preprint in perpetuity.

It is made available under a CC-BY 4.0 International license .

iSearch

Date searched: $7 / 5 / 2021$

Number of results: 851

Date filter: January 1, 2020 to present

Other filters applied: Search only title, abstract, preprints only

1. ("long COVID" OR "long covid-19" OR "long-covid" OR "long-covid-19" OR "long haul" OR "long hauler" OR "long haulers" OR "long-haul" OR "long-hauler" OR "long-haulers" OR "chronic COVID" OR "chronic covid-19" OR "post-acute COVID" OR "post-acute covid-19" OR "post acute COVID" OR "post acute covid-19" OR "persistent COVID" OR "persistent covid-19" OR "postCOVID" OR "post-covid-19" OR "post COVID" OR "post covid-19" OR "sequela" OR "sequelae" OR "long-term" OR "long term" OR "covid syndrome" OR "covid-19 syndrome" OR "persistent symptom" OR "persistent symptoms" OR "PASC" OR "PACS" OR "PPCS" OR "post-acute" OR "post acute")

2. ("prevalent" OR "prevalence" OR "occurrence" OR "occurrences" OR "duration" OR "durations" OR "length" OR "lengths" OR "risk factor" OR "risk factors" OR ("risk" AND "factor") OR ("risk" AND "factors") OR "predict" OR "prediction" OR "predictions" OR "predicting" OR "predictive" OR "predictor" OR "predictors" OR "symptom" OR "symptoms" OR "define" OR "defining" OR "definition" OR "definitions" OR "follow up" OR "follow-up" OR "followed up")

1 AND 2 
medRxiv preprint doi: https://doi.org/10.1101/2021.11.15.21266377; this version posted November 16, 2021. The copyright holder for this preprint (which was not certified by peer review) is the author/funder, who has granted medRxiv a license to display the preprint in perpetuity. It is made available under a CC-BY 4.0 International license .

\section{eMethods 2. Sample size calculation}

The inclusion/exclusion of sample size in the main text screening was based on a power calculation for Binomial proportions with the formula below:

$$
n=\frac{Z^{2} P(1-P)}{d^{2}}
$$

where

n: sample size

$Z$ statistic for $95 \% \mathrm{Cl}: z=1.96$, which is the 97.5 percentile point of the standard normal distribution

Expected proportion: $\mathrm{P}=0.3$

Margin of error: $d=0.05$. 


\section{eMethods 3. Meta-analysis framework}

We used random effects model with logit transformation and the DerSimonian-Laird (DL) estimator for $\tau^{2}$. Thus, the pooled estimated prevalence of PASC $(\hat{\theta})$ is calculated as:

$$
\hat{\theta}=\frac{\sum_{i} \hat{\theta}_{i} \widehat{w}_{i}}{\sum_{i} \widehat{w}_{i}} \text { with } \widehat{w}_{i}=\left(\hat{\tau}_{D L}^{2}+\hat{\xi}_{i}\right)^{-1}
$$

where $i$ represents the $i$-th study, $i=1,2, \ldots, k$; $\hat{\theta}_{i}$ is the logit transformed prevalence $p_{i}$ in study i so that $\hat{\theta}_{i}=\operatorname{logit}\left(p_{i}\right)=\ln \left(\frac{p_{i}}{1-p_{i}}\right)$, and $\widehat{w}_{i}$ is the estimated weight for study $i$ using the inverse-variance method.

The total variance of study $i$ is the sum of within-study variability denoted by $\hat{\xi}_{i}$, and between-study variability calculated by the $\mathrm{DL}$ estimator $\hat{\tau}_{D L}{ }^{2}$. Therefore, $\widehat{w}_{i}$ equals to the inverse of the variance, i.e., $\widehat{w}_{i}=\left(\hat{\tau}_{D L}^{2}+\hat{\xi}_{i}\right)^{-1}$

The DL estimator is calculated, as is given below:

$$
\hat{\tau}_{D L}{ }^{2}=\max \left\{0 ; \frac{Q_{c}-(k-1)}{\sum_{i} \hat{v}_{i}-\frac{\sum_{i} \hat{v}_{i}{ }^{2}}{\sum_{i} \hat{v}_{i}}}\right\} \text { where } \hat{v}_{i}=\hat{\xi}_{i}^{-1}
$$


medRxiv preprint doi: https://doi.org/10.1101/2021.11.15.21266377; this version posted November 16, 2021. The copyright holder for this preprint (which was not certified by peer review) is the author/funder, who has granted medRxiv a license to display the preprint in perpetuity.

It is made available under a CC-BY 4.0 International license .

eMethods 4. Risk of bias assessment across included articles

Using the Joanna Briggs Institute $(\mathrm{JBI})$ tool $^{3}$, studies were evaluated across 9 common sources of bias in observational studies and given a score out of 9 to reflect how well each study handled these biases. The vast majority (31/40) included studies scored $6 / 9$ or $7 / 9$. Only one study received a perfect score, and our lowest score was $4 / 9$. Supplementary file Supplementary_RiskofBias.xIsx contains full results from the risk of bias assessment across the included studies.

Next, we discuss the types of bias that can be introduced in cross-sectional and cohort studies, which make up the majority of the study designs in this review. First, several studies gave COVID-19 cases optional access to a post-COVID clinic or follow-up, which may have resulted in self-selection of sicker individuals. Reporting of symptoms was at times documented through self-report, which has been evidenced to differ from doctor-diagnosed symptoms. ${ }^{2}$ Among studies whose target population was a mixture of hospitalized and non-hospitalized individuals, proportions of hospitalized to non-hospitalized patients varied, possibly biasing the results for this group. Misclassification bias may also be of concern. Patients admitted to the ICU are known to sometimes experience so-called Post-Intensive Care Syndrome (PICS), whose symptomatology is somewhat similar to that of PASC ${ }_{-}^{3}$ Further, some studies suggest that as many as $85 \%$ of PASC patients experience symptom resolution, only relapse at a later date, which could obscure the true proportion of individuals experiencing chronic PASC. ${ }^{4}$ 
eTable 1. PRISMA checklist

\begin{tabular}{|c|c|c|c|}
\hline $\begin{array}{l}\text { Section and } \\
\text { Topic }\end{array}$ & $\begin{array}{l}\text { Ite } \\
\text { m \# }\end{array}$ & Checklist item & $\begin{array}{l}\text { Location } \\
\text { where item is } \\
\text { reported a }\end{array}$ \\
\hline \multicolumn{4}{|c|}{ TITLE } \\
\hline Title & 1 & Identify the report as a systematic review. & 1 \\
\hline \multicolumn{4}{|c|}{ ABSTRACT } \\
\hline Abstract & 2 & $\begin{array}{l}\text { Background: Provide an explicit statement of the main objective(s) or } \\
\text { question(s) the review addresses. Methods: Specify the inclusion and } \\
\text { exclusion criteria for the review. Specify the information sources (e.g. } \\
\text { databases, registers) used to identify studies and the date when each } \\
\text { was last searched. Specify the methods used to assess risk of bias in } \\
\text { the included studies. Specify the methods used to present and } \\
\text { synthesise results. Results: Give the total number of included studies } \\
\text { and participants and summarise relevant characteristics of studies. } \\
\text { Present results for main outcomes, preferably indicating the number of } \\
\text { included studies and participants for each. If meta-analysis was done, } \\
\text { report the summary estimate and confidence/credible interval. If } \\
\text { comparing groups, indicate the direction of the effect (i.e., which group } \\
\text { is favoured). Discussion: Provide a brief summary of the limitations of } \\
\text { the evidence included in the review (e.g., study risk of bias, } \\
\text { inconsistency and imprecision). Provide a general interpretation of the } \\
\text { results and important implications. Other: Specify the primary source of } \\
\text { funding for the review. Provide the register name and registration } \\
\text { number. }\end{array}$ & $2-4$ \\
\hline \multicolumn{4}{|c|}{ INTRODUCTION } \\
\hline Rationale & 3 & $\begin{array}{l}\text { Describe the rationale for the review in the context of existing } \\
\text { knowledge. }\end{array}$ & $6-8$ \\
\hline Objectives & 4 & $\begin{array}{l}\text { Provide an explicit statement of the objective(s) or question(s) the } \\
\text { review addresses. }\end{array}$ & 8 \\
\hline \multicolumn{4}{|c|}{$\begin{array}{l}\text { METHODS } \\
\end{array}$} \\
\hline Eligibility criteria & 5 & $\begin{array}{l}\text { Specify the inclusion and exclusion criteria for the review and how } \\
\text { studies were grouped for the syntheses. }\end{array}$ & $\begin{array}{l}\text { 9, eMethods } 1 \text {, } \\
\text { eMethods } 2\end{array}$ \\
\hline $\begin{array}{l}\text { Information } \\
\text { sources }\end{array}$ & 6 & $\begin{array}{l}\text { Specify all databases, registers, websites, organisations, reference lists } \\
\text { and other sources searched or consulted to identify studies. Specify the } \\
\text { date when each source was last searched or consulted. }\end{array}$ & 8 \\
\hline Search strategy & 7 & $\begin{array}{l}\text { Present the full search strategies for all databases, registers and } \\
\text { websites, including any filters and limits used. }\end{array}$ & eMethods 1 \\
\hline $\begin{array}{l}\text { Selection } \\
\text { process }\end{array}$ & 8 & $\begin{array}{l}\text { Specify the methods used to decide whether a study met the inclusion } \\
\text { criteria of the review, including how many reviewers screened each } \\
\text { record and each report retrieved, whether they worked independently, } \\
\text { and if applicable, details of automation tools used in the process. }\end{array}$ & 9 \\
\hline $\begin{array}{l}\text { Data collection } \\
\text { process }\end{array}$ & 9 & $\begin{array}{l}\text { Specify the methods used to collect data from reports, including how } \\
\text { many reviewers collected data from each report, whether they worked } \\
\text { independently, any processes for obtaining or confirming data from } \\
\text { study investigators, and if applicable, details of automation tools used in } \\
\text { the process. }\end{array}$ & $\begin{array}{l}9-10, \text { eMethods } \\
1\end{array}$ \\
\hline Data items & $10 a$ & $\begin{array}{l}\text { List and define all outcomes for which data were sought. Specify } \\
\text { whether all results that were compatible with each outcome domain in }\end{array}$ & 9-10, eTable 2 \\
\hline
\end{tabular}




\section{eTable 1 Cont'd}

\begin{tabular}{|c|c|c|c|}
\hline \multirow[t]{3}{*}{$\begin{array}{l}\text { Section and } \\
\text { Topic }\end{array}$} & $\begin{array}{l}\text { Ite } \\
\text { m \# }\end{array}$ & Checklist item & $\begin{array}{l}\text { Location } \\
\text { where item is } \\
\text { reported }{ }^{a}\end{array}$ \\
\hline & & $\begin{array}{l}\text { each study were sought (e.g., for all measures, time points, analyses), } \\
\text { and if not, the methods used to decide which results to collect. }\end{array}$ & \\
\hline & $10 \mathrm{~b}$ & $\begin{array}{l}\text { List and define all other variables for which data were sought (e.g., } \\
\text { participant and intervention characteristics, funding sources). Describe } \\
\text { any assumptions made about any missing or unclear information. }\end{array}$ & $9-10$ \\
\hline $\begin{array}{l}\text { Study risk of } \\
\text { bias } \\
\text { assessment }\end{array}$ & 11 & $\begin{array}{l}\text { Specify the methods used to assess risk of bias in the included studies, } \\
\text { including details of the tool(s) used, how many reviewers assessed } \\
\text { each study and whether they worked independently, and if applicable, } \\
\text { details of automation tools used in the process. }\end{array}$ & 11 , eMethods 4 \\
\hline $\begin{array}{l}\text { Effect } \\
\text { measures }\end{array}$ & 12 & $\begin{array}{l}\text { Specify for each outcome the effect measure(s) (e.g., risk ratio, mean } \\
\text { difference) used in the synthesis or presentation of results. }\end{array}$ & 10 \\
\hline \multirow[t]{6}{*}{$\begin{array}{l}\text { Synthesis } \\
\text { methods }\end{array}$} & $13 a$ & $\begin{array}{l}\text { Describe the processes used to decide which studies were eligible for } \\
\text { each synthesis (e.g. tabulating the study intervention characteristics } \\
\text { and comparing against the planned groups for each synthesis (item } \\
\# 5) \text { ). }\end{array}$ & $9-10$ \\
\hline & $13 b$ & $\begin{array}{l}\text { Describe any methods required to prepare the data for presentation or } \\
\text { synthesis, such as handling of missing summary statistics, or data } \\
\text { conversions. }\end{array}$ & 10, eTable 2 \\
\hline & $13 \mathrm{c}$ & $\begin{array}{l}\text { Describe any methods used to tabulate or visually display results of } \\
\text { individual studies and syntheses. }\end{array}$ & $10-11$ \\
\hline & $13 d$ & $\begin{array}{l}\text { Describe any methods used to synthesize results and provide a } \\
\text { rationale for the choice(s). If meta-analysis was performed, describe the } \\
\text { model(s), method(s) to identify the presence and extent of statistical } \\
\text { heterogeneity, and software package(s) used. }\end{array}$ & $\begin{array}{l}\text { 10-11, } \\
\text { eMethods } 3\end{array}$ \\
\hline & $13 e$ & $\begin{array}{l}\text { Describe any methods used to explore possible causes of } \\
\text { heterogeneity among study results (e.g., subgroup analysis, meta- } \\
\text { regression). }\end{array}$ & $\begin{array}{l}\text { 10-11, } \\
\text { eMethods } 3\end{array}$ \\
\hline & $13 f$ & $\begin{array}{l}\text { Describe any sensitivity analyses conducted to assess robustness of } \\
\text { the synthesized results. }\end{array}$ & 11, eFigure 1 \\
\hline $\begin{array}{l}\text { Reporting bias } \\
\text { assessment }\end{array}$ & 14 & $\begin{array}{l}\text { Describe any methods used to assess risk of bias due to missing } \\
\text { results in a synthesis (arising from reporting biases). }\end{array}$ & 11, eFigure 2 \\
\hline $\begin{array}{l}\text { Certainty } \\
\text { assessment }\end{array}$ & 15 & $\begin{array}{l}\text { Describe any methods used to assess certainty (or confidence) in the } \\
\text { body of evidence for an outcome. }\end{array}$ & $\begin{array}{l}\text { 11, eMethods } 4 \\
\text { and eFigure } 2\end{array}$ \\
\hline \multicolumn{4}{|c|}{ RESULTS } \\
\hline \multirow[t]{2}{*}{ Study selection } & $16 a$ & $\begin{array}{l}\text { Describe the results of the search and selection process, from the } \\
\text { number of records identified in the search to the number of studies } \\
\text { included in the review, ideally using a flow diagram. }\end{array}$ & 11, Figure 1 \\
\hline & $16 b$ & $\begin{array}{l}\text { Cite studies that might appear to meet the inclusion criteria, but which } \\
\text { were excluded, and explain why they were excluded. }\end{array}$ & 19 \\
\hline $\begin{array}{l}\text { Study } \\
\text { characteristics }\end{array}$ & 17 & Cite each included study and present its characteristics. & $\begin{array}{l}\text { 11-12, Figure } \\
\text { 1, Table } 1\end{array}$ \\
\hline $\begin{array}{l}\text { Risk of bias in } \\
\text { studies }\end{array}$ & 18 & Present assessments of risk of bias for each included study. & eMethods 4 \\
\hline $\begin{array}{l}\text { Results of } \\
\text { individual } \\
\text { studies }\end{array}$ & 19 & $\begin{array}{l}\text { For all outcomes, present, for each study: (a) summary statistics for } \\
\text { each group (where appropriate) and (b) an effect estimate and its } \\
\text { precision (e.g. confidence/credible interval), ideally using structured }\end{array}$ & Figure 2 \\
\hline
\end{tabular}




\section{eTable 1 Cont'd}

It is made available under a CC-BY 4.0 International license.

\begin{tabular}{|c|c|c|c|}
\hline $\begin{array}{l}\text { Section and } \\
\text { Topic }\end{array}$ & $\begin{array}{l}\text { Ite } \\
\text { m \# }\end{array}$ & Checklist item & $\begin{array}{l}\text { Location } \\
\text { where item is } \\
\text { reported }{ }^{a}\end{array}$ \\
\hline & & tables or plots. & \\
\hline \multirow[t]{4}{*}{$\begin{array}{l}\text { Results of } \\
\text { syntheses }\end{array}$} & $20 a$ & $\begin{array}{l}\text { For each synthesis, briefly summarise the characteristics and risk of } \\
\text { bias among contributing studies. }\end{array}$ & $12-15$ \\
\hline & $20 \mathrm{~b}$ & $\begin{array}{l}\text { Present results of all statistical syntheses conducted. If meta-analysis } \\
\text { was done, present for each the summary estimate and its precision } \\
\text { (e.g. confidence/credible interval) and measures of statistical } \\
\text { heterogeneity. If comparing groups, describe the direction of the effect. }\end{array}$ & $\begin{array}{l}\text { 12-16, Figure } \\
2-3, \text { Table } 2 \\
\text { eFigures } 1,4-7\end{array}$ \\
\hline & $20 c$ & $\begin{array}{l}\text { Present results of all investigations of possible causes of heterogeneity } \\
\text { among study results. }\end{array}$ & $\begin{array}{l}\text { 12-15, } \\
\text { eFigures } 1,4,5\end{array}$ \\
\hline & $20 d$ & $\begin{array}{l}\text { Present results of all sensitivity analyses conducted to assess the } \\
\text { robustness of the synthesized results. }\end{array}$ & eFigure 1,5 \\
\hline $\begin{array}{l}\text { Reporting } \\
\text { biases }\end{array}$ & 21 & $\begin{array}{l}\text { Present assessments of risk of bias due to missing results (arising from } \\
\text { reporting biases) for each synthesis assessed. }\end{array}$ & eFigure 2 \\
\hline $\begin{array}{l}\text { Certainty of } \\
\text { evidence }\end{array}$ & 22 & $\begin{array}{l}\text { Present assessments of certainty (or confidence) in the body of } \\
\text { evidence for each outcome assessed. }\end{array}$ & $\begin{array}{l}\text { eMethods } 4 \\
\text { and eFigure } 2\end{array}$ \\
\hline \multicolumn{4}{|c|}{ DISCUSSION } \\
\hline \multirow[t]{4}{*}{ Discussion } & $23 a$ & $\begin{array}{l}\text { Provide a general interpretation of the results in the context of other } \\
\text { evidence. }\end{array}$ & $16-17$ \\
\hline & $23 b$ & Discuss any limitations of the evidence included in the review. & 19 , eMethods 4 \\
\hline & $23 c$ & Discuss any limitations of the review processes used. & 19 \\
\hline & $23 d$ & $\begin{array}{l}\text { Discuss implications of the results for practice, policy, and future } \\
\text { research. }\end{array}$ & $16-20$ \\
\hline \multicolumn{4}{|c|}{ OTHER INFORMATION } \\
\hline \multirow[t]{3}{*}{$\begin{array}{l}\text { Registration } \\
\text { and protocol }\end{array}$} & $24 a$ & $\begin{array}{l}\text { Provide registration information for the review, including register name } \\
\text { and registration number, or state that the review was not registered. }\end{array}$ & $\mathrm{N} / \mathrm{A}$ \\
\hline & $24 b$ & $\begin{array}{l}\text { Indicate where the review protocol can be accessed, or state that a } \\
\text { protocol was not prepared. }\end{array}$ & $\mathrm{N} / \mathrm{A}$ \\
\hline & $24 \mathrm{c}$ & $\begin{array}{l}\text { Describe and explain any amendments to information provided at } \\
\text { registration or in the protocol. }\end{array}$ & N/A \\
\hline Support & 25 & $\begin{array}{l}\text { Describe sources of financial or non-financial support for the review, } \\
\text { and the role of the funders or sponsors in the review. }\end{array}$ & 35 \\
\hline $\begin{array}{l}\text { Competing } \\
\text { interests }\end{array}$ & 26 & Declare any competing interests of review authors. & 35 \\
\hline $\begin{array}{l}\text { Availability of } \\
\text { data, code and } \\
\text { other materials }\end{array}$ & 27 & $\begin{array}{l}\text { Report which of the following are publicly available and where they can } \\
\text { be found: template data collection forms; data extracted from included } \\
\text { studies; data used for all analyses; analytic code; any other materials } \\
\text { used in the review. }\end{array}$ & $\mathrm{N} / \mathrm{A}$ \\
\hline
\end{tabular}

${ }^{\mathrm{a}} \mathrm{N} / \mathrm{A}=$ not applicable. 
eTable 2. Characterization and synonyms of symptoms

\begin{tabular}{|c|c|}
\hline Symptom & Characterization and synonyms \\
\hline Fatigue & $\begin{array}{l}\text { fatigue, felt tired, lassitude/fatigue, malaise and fatigue, malaise or fatigu, persistent } \\
\text { fatigue }\end{array}$ \\
\hline Cough & cough, dry cough, persistent cough, persistent dry cough \\
\hline Dyspnea & $\begin{array}{l}\text { breathlessness, dyspnea, dyspnoea/shortness of breath, exertional dyspnea, } \\
\text { problems breathing, shortness of breath, difficulty breathing, dysponea, shortness } \\
\text { of breath/breathlessness }\end{array}$ \\
\hline Headache & headache, heachache \\
\hline Smell & $\begin{array}{l}\text { anosmia, loss of smell, reduce sense of smell, hyposmia, alterations of smell, smell } \\
\text { disorder }\end{array}$ \\
\hline Taste & aguesia, dysgeusia, dysguesia, loss of taste, taste disorder, alteration of taste \\
\hline Smell/Taste & $\begin{array}{l}\text { altered sense of smell or taste, anosmia or aguesia, anosmia-dysgeusia, } \\
\text { anosmia/ageusia, anosmia/aguesia, diminished taste/smell, loss of mell/taste, } \\
\text { problems with taste or smell, smell or taste disorder, anosmia/dysgeusia, altered } \\
\text { smell or taste, taste/smell, loss of taste/smel, anosmia and/or aguesia }\end{array}$ \\
\hline Anxiety & anxiety, feeling anxious, symptoms of anxiety \\
\hline Depression & depression, feeling depressed, depressive, depressed mood \\
\hline Fever & Fever \\
\hline Myalgia & $\begin{array}{l}\text { myalgia, arthralgia, myalgia or arthralgia, myalgias-arthralgias, myalgias, } \\
\text { myalgia/arthralgias, unusual muscle pains, muscle aches, muscle pain, muscle } \\
\text { aches, persistent muscle pain, muscle aches (myalgia) }\end{array}$ \\
\hline Hair loss & alopecia, hair loss, loss hair \\
\hline Insomnia & insomia, insomnia, sleep disorder, sleeping disturbances, sleeplessness, \\
\hline Joint pain & joint pain, articular pains \\
\hline Sore throat & Sore throat, pharyngodynia, throat itching, throat pain \\
\hline Chest pain & chest pain, chest pains, nonspecific chest pain \\
\hline Abdominal pain & abdominal pain \\
\hline Mood disorders & mood disorders, mood changes, mood disturbances \\
\hline Diarrhea & Diarrhea, diarrhoea \\
\hline Digestive & digestive symptoms \\
\hline Dizziness & Dizziness \\
\hline $\begin{array}{l}\text { Concentration/ } \\
\text { Confusion/Brain fog }\end{array}$ & $\begin{array}{l}\text { Confusion, lack of concentration, attention disorders, concentration, concentration } \\
\text { problems, confusion/lack of concentration, impairment (brain fog, loss of } \\
\text { concentration), inability to concentrate, problems concentrating and thinking, brain } \\
\text { fog }\end{array}$ \\
\hline Appetite/eating disorder & $\begin{array}{l}\text { appetite, anorexia, decreased appetite, decreased or lack of appetite, eating } \\
\text { disorders, loss of appetite }\end{array}$ \\
\hline Tachycardia & tachycardia, tachycardia-palpitations, palpitations \\
\hline
\end{tabular}

Symptoms names were inconsistent across studies. Therefore, we grouped symptoms into groups according to similarity as detailed in eTable 2. 
medRxiv preprint doi: https://doi.org/10.1101/2021.11.15.21266377; this version posted November 16, 2021. The copyright holder for this preprint (which was not certified by peer review) is the author/funder, who has granted medRxiv a license to display the preprint in perpetuity.

It is made available under a CC-BY 4.0 International license .

eTable 3. Summary of excluded articles by region and follow-up time a, b

\begin{tabular}{|c|c|c|c|c|c|c|c|c|}
\hline $\begin{array}{l}\text { Exclusion } \\
\text { Reason } \\
\end{array}$ & Africa & Asia & Australia & Europe & $\begin{array}{l}\text { North } \\
\text { America }\end{array}$ & $\begin{array}{l}\text { South } \\
\text { America }\end{array}$ & Mix & Total \\
\hline Abstract only & & & 1 & 8 & 8 & & & 17 \\
\hline Duplicate cohort & & & & 1 & & & & 1 \\
\hline Duplicated & & & & & & & & 0 \\
\hline $\begin{array}{l}\text { Wrong Follow- } \\
\text { up Time }\end{array}$ & 4 & 6 & & 9 & 2 & & 1 & 22 \\
\hline $\begin{array}{l}\text { Wrong Index } \\
\text { Date }\end{array}$ & 1 & & & 1 & & & & 2 \\
\hline Sample Size & 2 & 7 & 1 & 38 & 11 & 1 & 1 & 61 \\
\hline Wrong Outcome & & 8 & & 28 & 16 & & 4 & 56 \\
\hline $\begin{array}{l}\text { Wrong } \\
\text { Population }\end{array}$ & & 1 & & 14 & 6 & 1 & 4 & 26 \\
\hline $\begin{array}{l}\text { Wrong } \\
\text { Publication Type }\end{array}$ & & & & 4 & 1 & & & 5 \\
\hline Total & 7 & 22 & 2 & 103 & 44 & 2 & 10 & 190 \\
\hline
\end{tabular}

a Studies excluded during the full-text screening are represented in this table.

${ }^{b}$ Studies where region is not applicable or unknown are not represented in this table 
medRxiv preprint doi: https://doi.org/10.1101/2021.11.15.21266377; this version posted November 16, 2021. The copyright holder for this preprint (which was not certified by peer review) is the author/funder, who has granted medRxiv a license to display the preprint in perpetuity.

It is made available under a CC-BY 4.0 International license .

eTable 4. Summary of significant non-meta-analyzed risk factors for PASC

\begin{tabular}{|c|c|c|c|c|}
\hline Studies & Predictor & $\begin{array}{l}\text { Odds Ratio } \\
\text { (OR) }\end{array}$ & $\begin{array}{l}95 \% \text { lower } \\
\text { confidence } \\
\text { bound }\end{array}$ & $\begin{array}{l}95 \% \text { upper } \\
\text { confidence } \\
\text { bound }\end{array}$ \\
\hline Augustin et al. ${ }^{5}$ & $\begin{array}{l}\text { Number of symptoms in acute } \\
\text { COVID-19 }\end{array}$ & 1.29 & 1.08 & 1.55 \\
\hline \multirow[t]{2}{*}{ Naik et al. ${ }^{6}$} & Hypothyroidism & 4.13 & 2.20 & 7.60 \\
\hline & $\begin{array}{l}\text { COVID-19 severity: moderate } \\
\text { vs mild }\end{array}$ & 1.70 & 1.10 & 2.40 \\
\hline \multirow[t]{2}{*}{ Menges et al. ${ }^{7}$} & $\begin{array}{l}\text { Severe to very severe initial } \\
\text { symptoms (mild to moderate } \\
\text { reference group) }\end{array}$ & 2.05 & 1.27 & 3.34 \\
\hline & Comorbidities & 2.08 & 1.24 & 3.50 \\
\hline \multirow[t]{6}{*}{ Perlis et al. ${ }^{8}$} & $\begin{array}{l}\text { Covid severity: somewhat vs } \\
\text { not at all }\end{array}$ & 2.59 & 1.75 & 3.94 \\
\hline & $\begin{array}{l}\text { Covid severity: very vs not at } \\
\text { all }\end{array}$ & 3.78 & 2.54 & 5.79 \\
\hline & Muscle pain & 1.31 & 1.03 & 1.67 \\
\hline & Headache & 1.44 & 1.11 & 1.86 \\
\hline & Shaking & 1.3 & 1.01 & 1.66 \\
\hline & Age (decade) & 1.1 & 1.02 & 1.2 \\
\hline \multirow[t]{7}{*}{ Yomogida et al. ${ }^{10}$} & Pre-existing condition & 2.14 & 1.33 & 3.43 \\
\hline & Asthma & 4.50 & 2.12 & 9.57 \\
\hline & Obesity & 7.73 & 2.51 & 23.83 \\
\hline & Hypertension & 1.86 & 1.09 & 3.18 \\
\hline & $\begin{array}{l}\text { Severe pre-existing conditions } \\
\text { (stroke, cancer, } \\
\text { immunocompromising } \\
\text { conditions, liver, lung, and } \\
\text { kidney disorders) }\end{array}$ & 2.46 & 1.14 & 5.30 \\
\hline & $\begin{array}{l}\text { Severe Covid at diagnosis } \\
\text { (asymptomatic reference } \\
\text { group) }\end{array}$ & 13.33 & 2.43 & 73.02 \\
\hline & $\begin{array}{l}\text { Moderate Covid at diagnosis } \\
\text { (asymptomatic reference } \\
\text { group) }\end{array}$ & 12.75 & 2.86 & 56.88 \\
\hline
\end{tabular}


medRxiv preprint doi: https://doi.org/10.1101/2021.11.15.21266377; this version posted November 16, 2021. The copyright holder for this preprint (which was not certified by peer review) is the author/funder, who has granted medRxiv a license to display the preprint in perpetuity.

\section{eTable 4 Cont'd}

It is made available under a CC-BY 4.0 International license .

\begin{tabular}{|l|l|c|c|c|}
\hline Studies & Predictor & $\begin{array}{l}\text { Odds Ratio } \\
\text { (OR) }\end{array}$ & $\begin{array}{l}\text { 95\% lower } \\
\text { confidence } \\
\text { bound }\end{array}$ & $\begin{array}{l}\text { 95\% upper } \\
\text { confidence } \\
\text { bound }\end{array}$ \\
\hline \multirow{5}{*}{} & $\begin{array}{l}\text { Mild Covid at diagnosis } \\
\text { (asymptomatic reference } \\
\text { group) }\end{array}$ & 5.00 & 1.51 & 21.77 \\
\cline { 2 - 5 } & $\begin{array}{l}\text { Age 40-54 (25-39 reference } \\
\text { group) }\end{array}$ & 1.81 & 1.05 & 3.12 \\
\hline \multirow{3}{*}{ Sudre et al. ${ }^{11}$} & Fatigue in first week & 2.83 & 2.09 & 3.83 \\
\cline { 2 - 5 } & Headache in first week & 2.62 & 2.04 & 3.37 \\
\cline { 2 - 5 } & Dyspnea in first week & 2.36 & 1.91 & 2.91 \\
\cline { 2 - 5 } & Hoarse voice in first week & 2.33 & 1.88 & 2.90 \\
\cline { 2 - 5 } & Myalgia in first week & 2.22 & 1.80 & 2.73 \\
\hline \multirow{2}{*}{ Desgranges ${ }^{12}$} & Female & 1.67 & 1.09 & 2.56 \\
\cline { 2 - 5 } & Overweight/obese & 1.67 & 1.10 & 2.56 \\
\hline
\end{tabular}

eTable 4 presents a list of non-meta-analyzed risk factors and their associated odds ratios and confidence intervals. Only factors with a significant positive association with PASC were included (i.e., $\mathrm{OR}>1$ and $\mathrm{Cl}$ does not cross 1 ). If both odds ratios and adjusted odds ratios were provided for a risk factor, we used the adjusted odds ratio. 
medRxiv preprint doi: https://doi.org/10.1101/2021.11.15.21266377; this version posted November 16, 2021. The copyright holder for this preprint (which was not certified by peer review) is the author/funder, who has granted medRxiv a license to display the preprint in perpetuity.

It is made available under a CC-BY 4.0 International license .

eTable 5. Summary of duration of PASC and symptoms

\begin{tabular}{|l|c|c|c|}
\hline \multirow{2}{*}{ Outcome } & \multicolumn{3}{|c|}{$\begin{array}{l}\text { Median duration of PASC or symptoms } \\
\text { by studies [IQR] }\end{array}$} \\
\hline & \multicolumn{2}{|c|}{ Sudre et al. ${ }^{11}$} & Naik et al. ${ }^{6}$ \\
\hline & $28+$ days & $56+$ days & 28 days \\
\hline PASC & 41 & & \\
\hline Abdominal pain & 7 & 13 & \\
\hline Chest pain & 13 & 46 & $60[41-112]$ \\
\hline Cough & 20 & 34 & $60[45-118]$ \\
\hline Delirium & 8 & 14 & \\
\hline Dyspnea & 24 & 59 & $90[45-124]$ \\
\hline Fatigue & 33 & 73 & $60[45-135]$ \\
\hline Headache & 22 & 56 & \\
\hline Myalgia & 7 & 30 & $60[45-150]$ \\
\hline Loss of smell & 24 & 53 & \\
\hline Sore throat & 15 & 33 & \\
\hline Fever & 6 & 11 & \\
\hline Disturbed sleep & & & $45[40-70]$ \\
\hline
\end{tabular}

Summary of two studies that evaluated median duration of PASC and specific symptoms. Sudre et al further calculated median duration by different follow-up time, at least 28 days after index date and at least 56 days after the index date. 
medRxiv preprint doi: https://doi.org/10.1101/2021.11.15.21266377; this version posted November 16, 2021. The copyright holder for this preprint (which was not certified by peer review) is the author/funder, who has granted medRxiv a license to display the preprint in perpetuity.

It is made available under a CC-BY 4.0 International license .

\section{eFigure 1. Stratified analysis of PASC by persisted versus not persisted symptoms}

$\begin{array}{lc}\text { Studies } & \text { Prevalence }(95 \% \\ \text { persistent = not recovered/at least one symptom } \\ \text { Huang et al China } & 0.76[0.74 ; 0.78] \\ \text { Wong-Chew et al Mexico } & 0.76[0.74 ; 0.78] \\ \text { Areekal et al India } & 0.66[0.61 ; 0.71] \\ \text { Lemhofer et al Germany } & 0.62[0.57 ; 0.67] \\ \text { Munblit et al Russia } & 0.58[0.56 ; 0.60] \\ \text { Maestre-Muñiz et al Spain } & 0.57[0.53 ; 0.61] \\ \text { Zheng et al China } & 0.56[0.52 ; 0.60] \\ \text { Desgranges et al Switzerland } & 0.53[0.48 ; 0.58] \\ \text { Venturelli et al Italy } & 0.51[0.48 ; 0.55] \\ \text { Morin et al France } & 0.51[0.46 ; 0.56] \\ \text { Xiong et al China } & 0.50[0.45 ; 0.54] \\ \text { Yomogida et al USA } & 0.48[0.43 ; 0.53] \\ \text { Budhiraja et al India } & 0.40[0.37 ; 0.43] \\ \text { Peghin et al Europe } & 0.40[0.36 ; 0.44] \\ \text { Righi et al Europe } & 0.39[0.35 ; 0.44] \\ \text { Cirulli et al USA } & 0.36[0.31 ; 0.41] \\ \text { Shang et al China } & 0.33[0.30 ; 0.36] \\ \text { Spotnitz et al USA } & 0.28[0.27 ; 0.28] \\ \text { Menges et al Switzerland } & 0.26[0.22 ; 0.30] \\ \text { Shoucri et al USA } & 0.25[0.22 ; 0.30] \\ \text { Naik et al } \square \text { India } & 0.22[0.20 ; 0.24] \\ \text { Sudre et al UK/SE/US } & 0.13[0.12 ; 0.14] \\ \text { Perlis et al USA } & 0.09[0.08 ; 0.10] \\ \text { Total } & 0.43[0.34 ; 0.52] \\ \text { Heterogeneity: } \chi_{22}^{2}=6273.22(P<.001), I^{2}=100 \% \\ \text { persistent = persistent } & \\ \text { Fernández-de-Las-Peñas et al Spain } & 0.81[0.79 ; 0.84] \\ \text { Ghosn et al France } & 0.68[0.65 ; 0.71] \\ \text { Hirschtick et al USA } & 0.52[0.48 ; 0.57] \\ \text { Munblit et al Russia } & 0.47[0.45 ; 0.49] \\ \text { Augustin et al Europe } & 0.28[0.24 ; 0.32] \\ \text { Huang et al California } & 0.27[0.25 ; 0.30] \\ \text { Lampl et al Germany } & 0.09[0.06 ; 0.12] \\ \text { Total } & 0.43[0.27 ; 0.60] \\ \text { Heterogeneity: } \chi_{6}^{2}=1042.78(P<.001), I^{2}=99 \% \\ \text { Total } & 0.43 ; 0.51] \\ \text { Heterogeneity: } \chi_{29}^{2}=8543.78(P<.001) & I^{2}=100 \% \\ & \end{array}$

Study population

$\square$ Not Hospitalized

$\square$ Hospitalized and Non-Hospitalized Mix

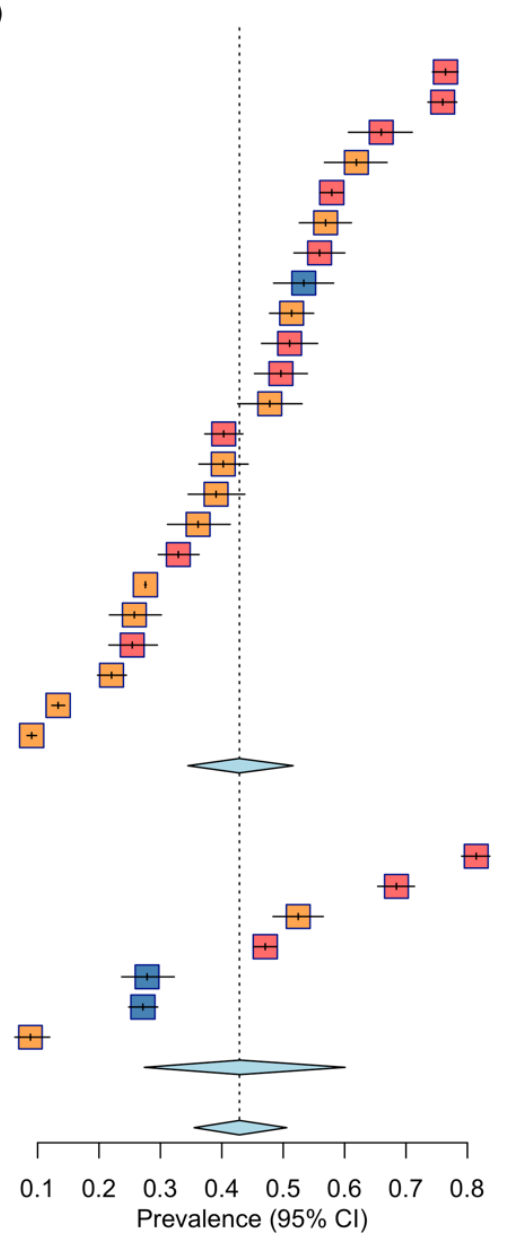

PASC definitions varied across studies. eFigure 1 stratified PASC prevalence according to whether a study defined PASC as having at least one symptom or not recovered at follow-up, or having persistent symptoms at follow-up. 


\section{eFigure 2. Funnel plot for publication bias assessment among included studies}

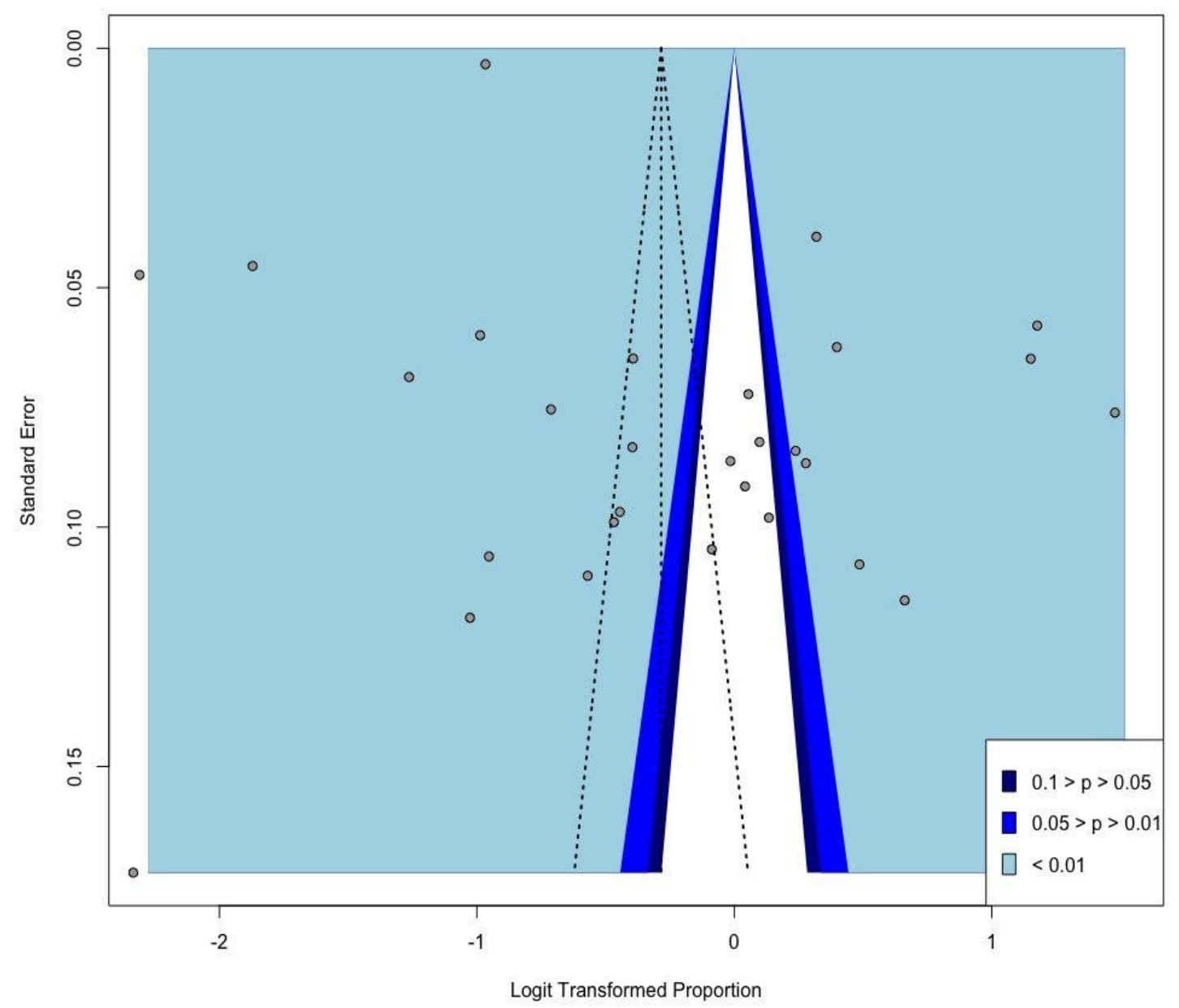

eFigure 2 presents the funnel plot for examination of publication bias among the included studies in the quantitative synthesis. The standard procedure of an initial visual inspection for publication bias suggests that asymmetry may be present. In further formally testing for presence of asymmetry, the Egger's test statistic of 3.18 is significant ( $p$-value of $0.003<0.05$ ), and as a further check, the Begg rank correlation test is performed with a resulting test statistic that is not significant ( $p$-value of $0.679>0.05$ ). The contrasting results is generally not uncommon, as the concordance between these two tests has been found to be moderate. ${ }^{4}$ Moreover, the Begg test has been evidenced to result in larger $p$-values for meta-analyses without a considerably number of studies of which the synthesis herein may qualify (i.e., number of included studies is 29; threshold for small meta-analyses is 25 studies, whereas roughly 75 studies constitute a large meta-analysis). ${ }^{13}$ In sum, although the Egger's test indicates a detection of bias from publishing, for meta-analyzing proportion estimates from observational studies, asymmetry in this context does not strictly indicate publication bias as proportions are published without preference over a particular effect size ${ }^{5}$. Additionally, with most included articles being hospital-based or multi-center study designs, the results may be driven by the larger sample sizes. Additionally, we suspect that heterogeneity in the true effect sizes of PASC prevalence, across the geographic entities reflected in the included studies, accounts for some of the horizontal spread in the proportion estimates ${ }^{5}$. 
medRxiv preprint doi: https://doi.org/10.1101/2021.11.15.21266377; this version posted November 16, 2021. The copyright holder for this preprint (which was not certified by peer review) is the author/funder, who has granted medRxiv a license to display the preprint in perpetuity.

It is made available under a CC-BY 4.0 International license .

\section{eFigure 3. PRISMA flow diagram for extended search}
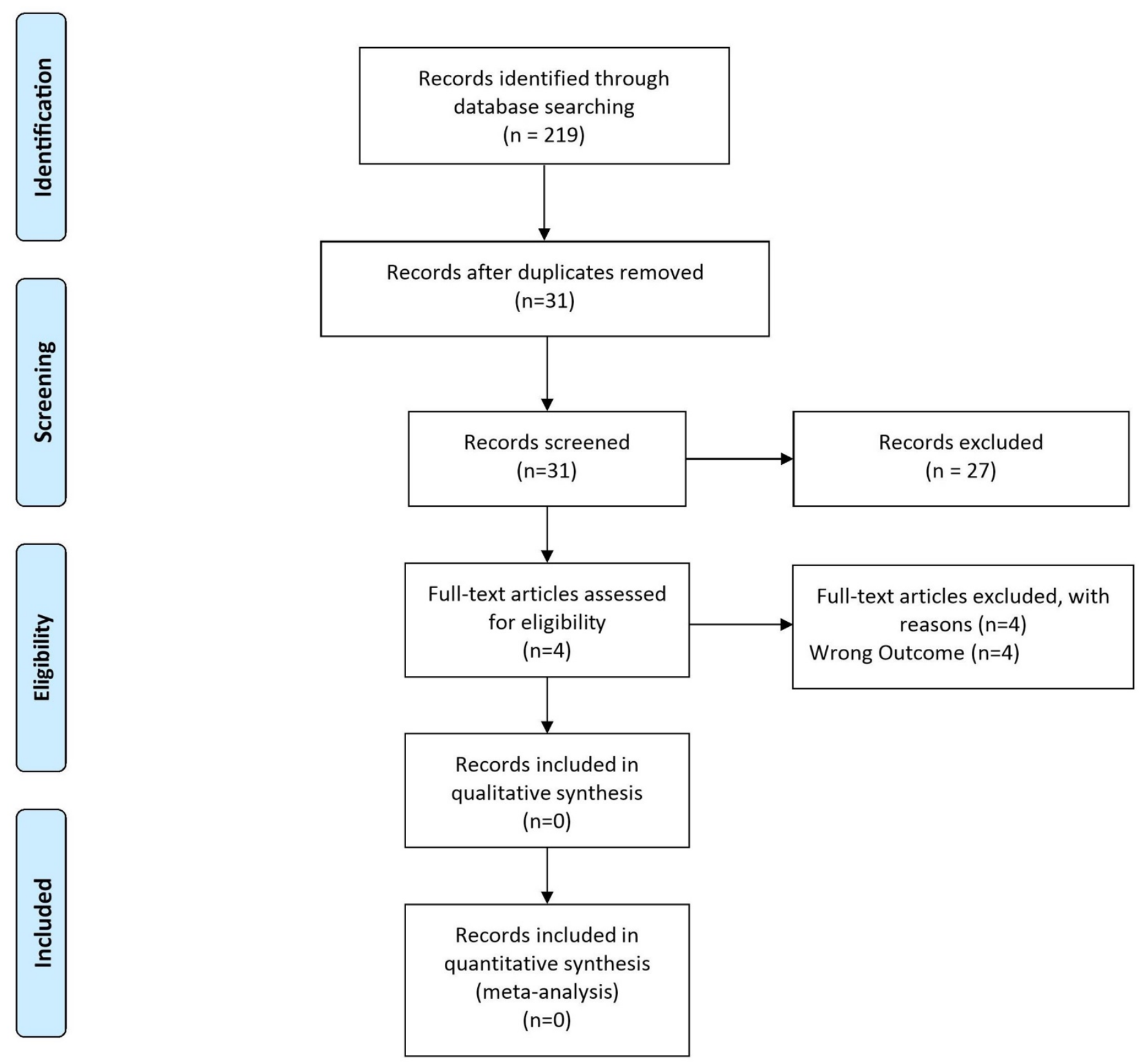

eFigure 3 details the study selection process for the search extension we performed on August 12, 2021 as described in Supplement B. We found 31 new studies (not covered in the original search) from selected journals, which we then screened. None of these studies met our inclusion/exclusion criteria, and thus none were included in the systematic review or meta-analysis. 
medRxiv preprint doi: https://doi.org/10.1101/2021.11.15.21266377; this version posted November 16, 2021. The copyright holder for this preprint (which was not certified by peer review) is the author/funder, who has granted medRxiv a license to display the preprint in perpetuity.

It is made available under a CC-BY 4.0 International license .

eFigure 4. Forest plots with the prevalence estimates of PASC stratified by (A) gender (B) hospitalization, (C) region, and (D) follow-up time, respectively
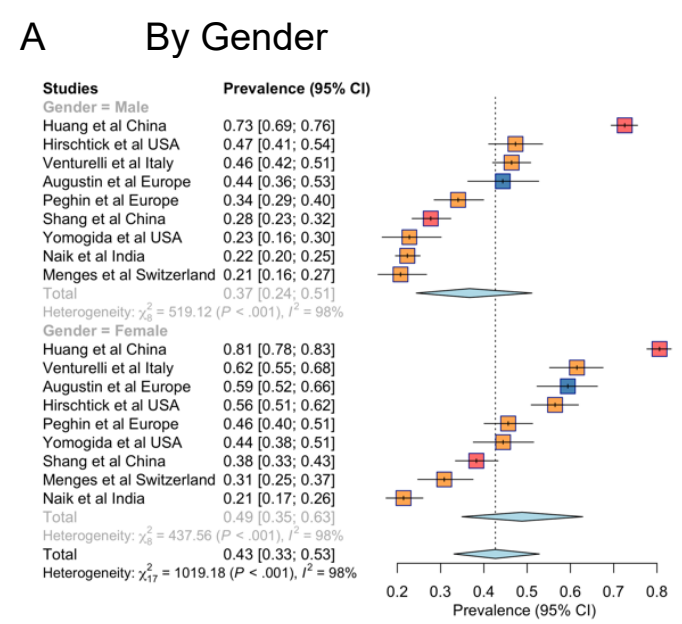

C

By Region
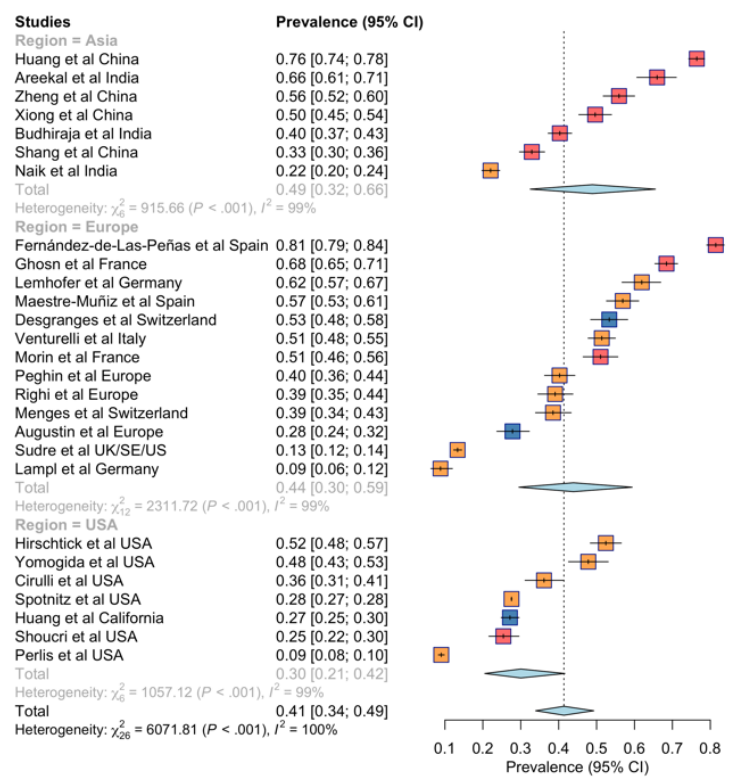

B By Hospitalization

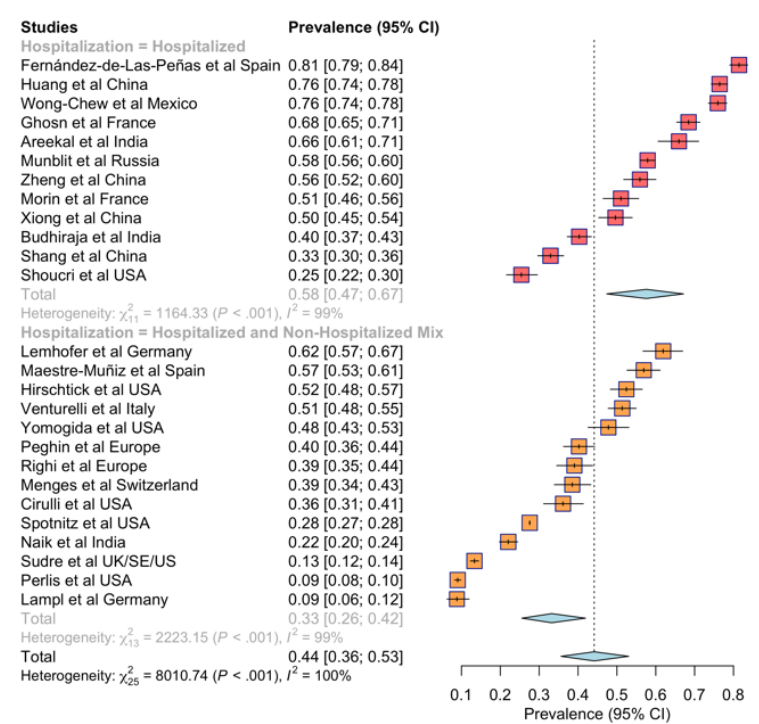

D

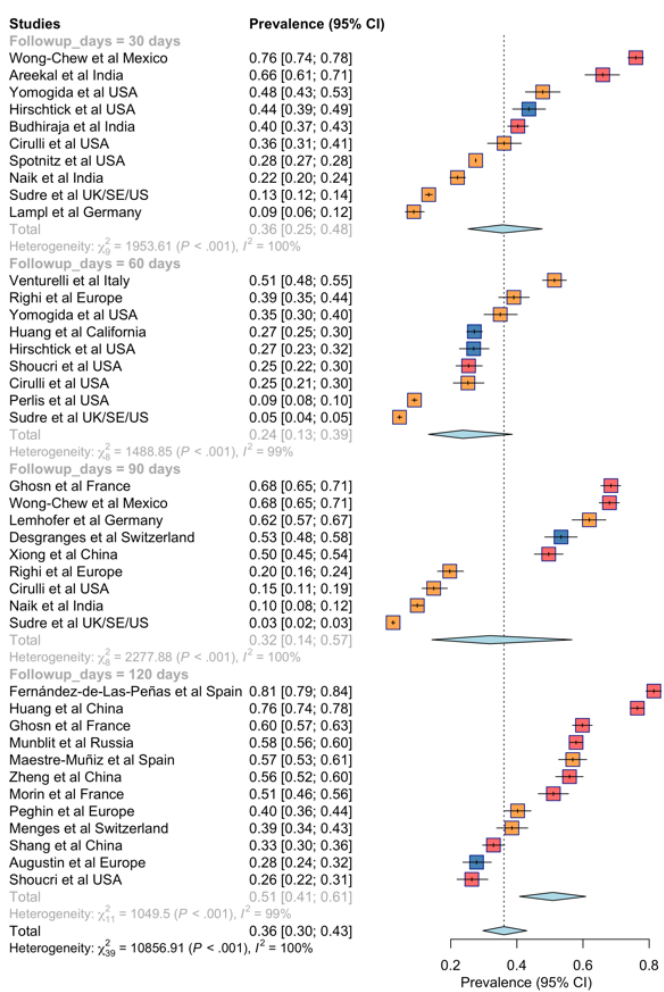

eFigure 4 reports stratified meta-analysis by gender, hospitalization, region, and follow-up time. The legend is consistent across all subfigures where blue, orange, red represents studies with non-hospitalized population, mixed of hospitalized and non-hospitalized population, and hospitalized population respectively.

Study population

$\square$ Not Hospitalized

Hospitalized and Non-Hospitalized Mix

$\square$ Hosvitalized 
medRxiv preprint doi: https://doi.org/10.1101/2021.11.15.21266377; this version posted November 16, 2021. The copyright holder for this preprint (which was not certified by peer review) is the author/funder, who has granted medRxiv a license to display the preprint in perpetuity.

It is made available under a CC-BY 4.0 International license .

eFigure 5. Meta-analysis of studies with 120 follow-up days stratified by acute-phase hospitalization status of study population

a) Mixed of hospitalized and non-hospitalized individuals

Studies Prevalence $(95 \% \mathrm{Cl})$

Maestre-Muñiz et al Spain 0.57 [0.53; 0.61]

Peghin et al Europe $0.40[0.36 ; 0.44]$

Menges et al Switzerland $0.39[0.34 ; 0.43]$

Total $0.45[0.34 ; 0.57]$

Heterogeneity: $\chi_{2}^{2}=43.09(P<.001), I^{2}=95 \%$

Study population

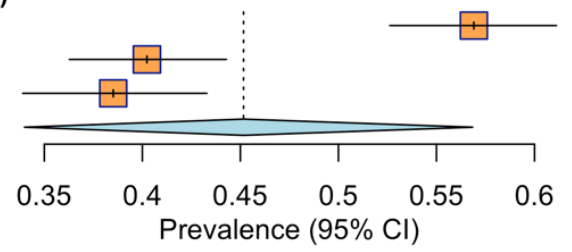

$\square$ Not Hospitalized

$\square$ Hospitalized and Non-Hospitalized Mix

$\square$ Hospitalized

b) Hospitalized individuals

Studies Prevalence $(95 \% \mathrm{Cl})$

Fernández-de-Las-Peñas et al Spain 0.81 [0.79; 0.84]

Huang et al China $\quad 0.76[0.74 ; 0.78]$

Ghosn et al France $\quad 0.60[0.57 ; 0.63]$

Munblit et al Russia $\quad 0.58[0.56 ; 0.60]$

Zheng et al China $\quad 0.56[0.52 ; 0.60]$

Morin et al France $\quad 0.51[0.46 ; 0.56]$

Shang et al China $\quad 0.33[0.30 ; 0.36]$

Shoucri et al USA $0.26[0.22 ; 0.31]$

Total $\quad 0.56[0.44 ; 0.68]$

Heterogeneity: $\chi_{7}^{2}=765.15(P<.001), I^{2}=99 \%$

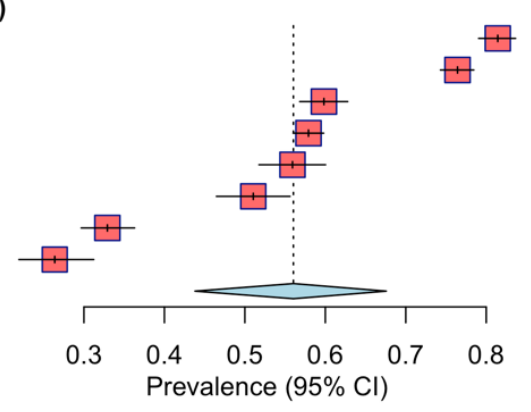

Study population

ot Hospitalized

$\square$ Hospitalized and Non-Hospitalized Mix

$\square$ Hosbitalized 
medRxiv preprint doi: https://doi.org/10.1101/2021.11.15.21266377; this version posted November 16, 2021. The copyright holder for this preprint (which was not certified by peer review) is the author/funder, who has granted medRxiv a license to display the preprint in perpetuity.

\section{eFigure 6 Cont'd}

\section{eFigure 6. Supplementary meta-analysis of PASC symptom-specific prevalence}

Fatigue

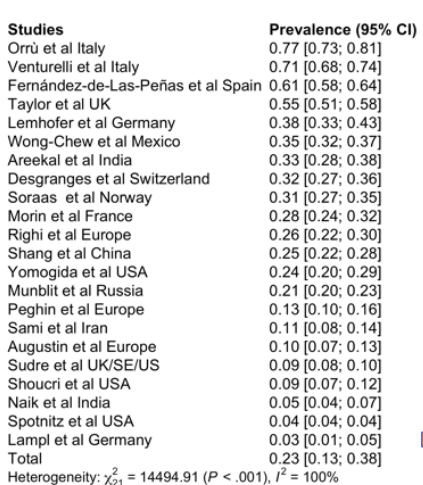

Insomia

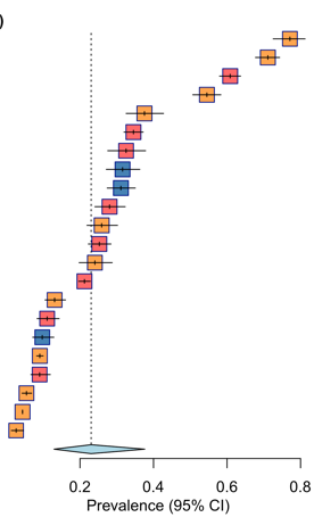

Studies
Taylor et al UK Mannan et al Bangladesh Lemhofer et al Germany Zheng et al China Huang et al China Shang et al China
Wong-Chew et al Mexico Wong-Chew et al
Xiong et al China Munblit et al Russia Areekal et al India
Sami et al Iran Spolniz et alUSA Naik et al Ind Prevalence $(95 \% \mathrm{Cl})$ $0.40[0.36 ; 0.44]$ $0.31[0.28 ; 0.33]$ $0.30[0.25 ; 0.35]$ $0.26[0.24 ; 0.29$ $0.23[0.20 ; 0.26]$ $0.18[0.15 ; 0.21]$ $0.10[0.07 ; 0.13$ $0.07[0.04 ; 0.10$ $0.06[0.04 ; 0.08$ $0.02[0.02 ; 0.02$ $0.13[0.06 ; 0.28$ Heterogeneity: $x_{13}^{2}=9378.13(P<.001), I^{2}=100 \%$
Dyspnea

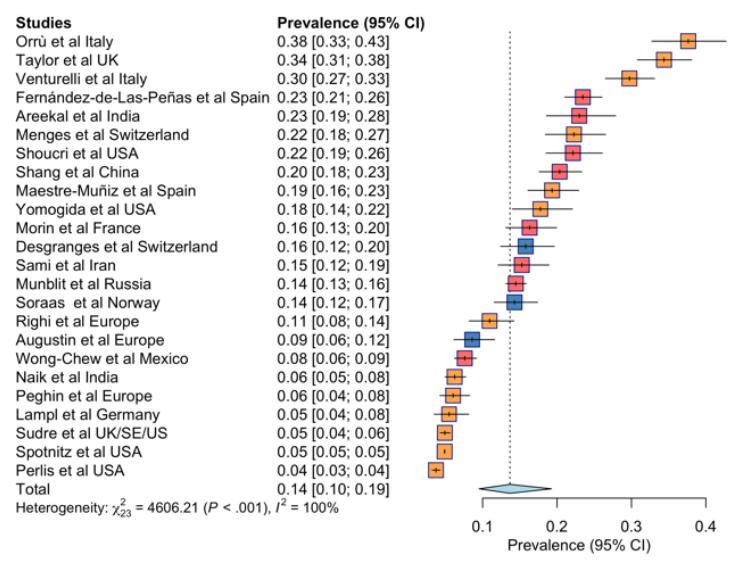

Joint pain

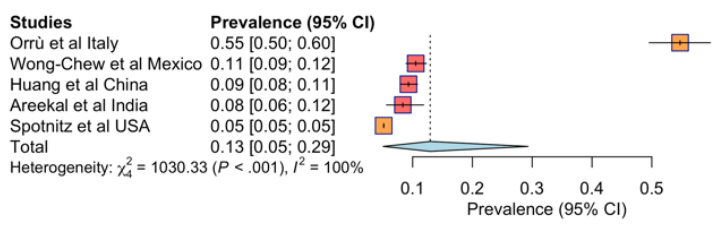


medRxiv preprint doi: https://doi.org/10.1101/2021.11.15.21266377; this version posted November 16, 2021. The copyright holder for this preprint (which was not certified by peer review) is the author/funder, who has granted medRxiv a license to display the preprint in perpetuity.

\section{eFigure 6 Cont'd}

Memory problems

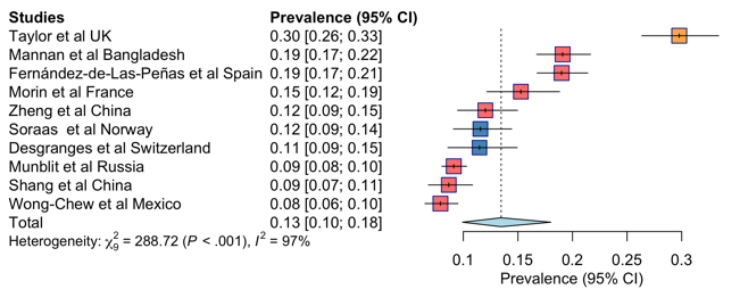

Depression
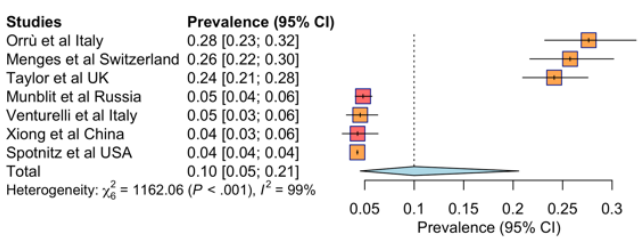

Smell
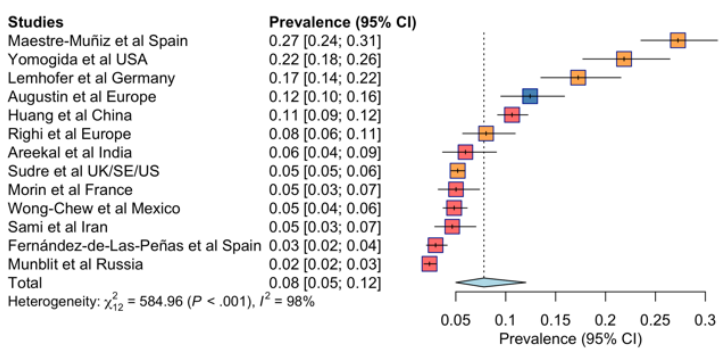

Anxiety

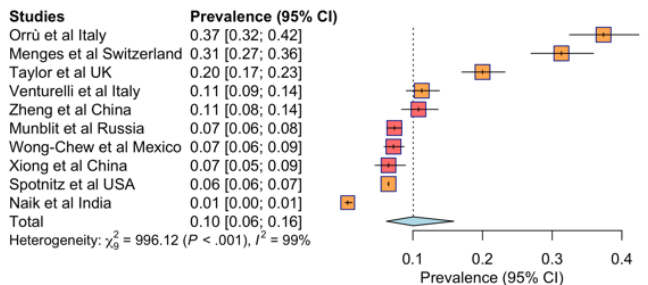

Concentration or Confusion or Brain fog

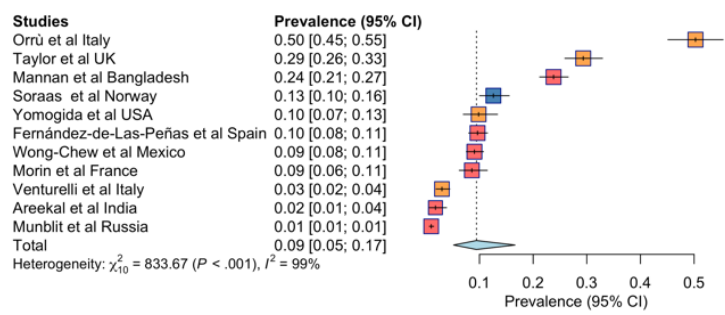

Taste

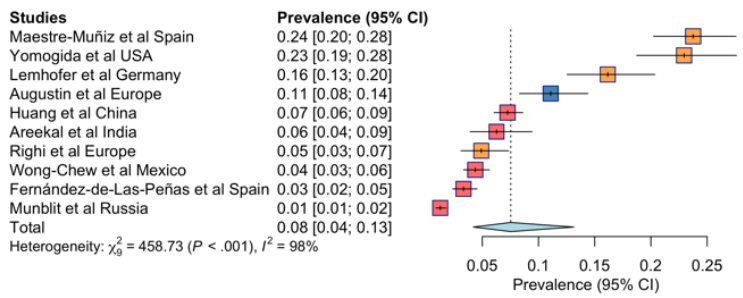


medRxiv preprint doi: https://doi.org/10.1101/2021.11.15.21266377; this version posted November 16, 2021. The copyright holder for this preprint (which was not certified by peer review) is the author/funder, who has granted medRxiv a license to display the preprint in perpetuity.

\section{eFigure 6 Cont'd}

It is made available under a CC-BY 4.0 International license .

\section{Cough}

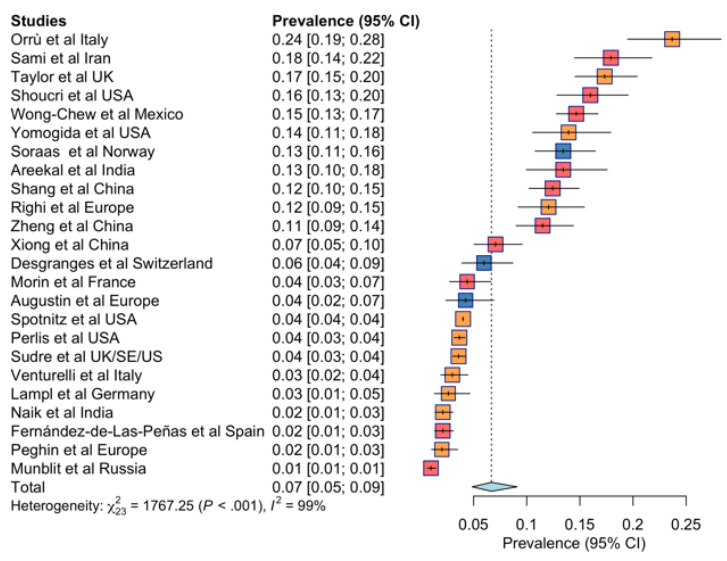

Tachycardia

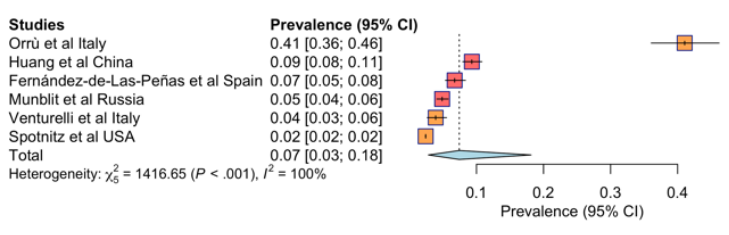

Dizziness

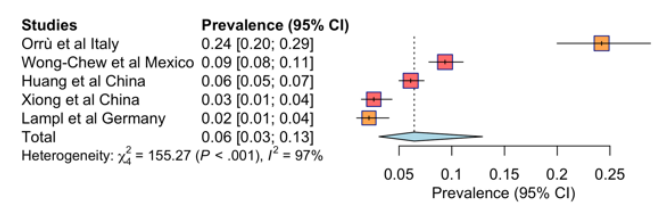

Hair loss

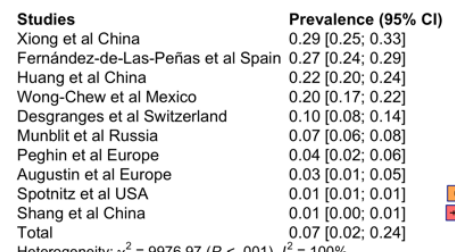

$\begin{array}{ll}\text { Total } & 0.07[0.02 ; 0.24] \\ & \end{array}$

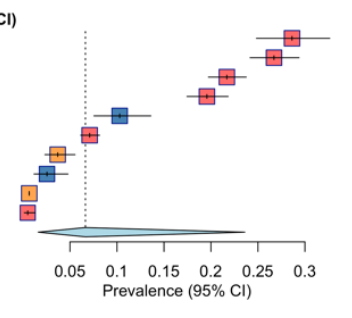

Appetite or Eating disorder

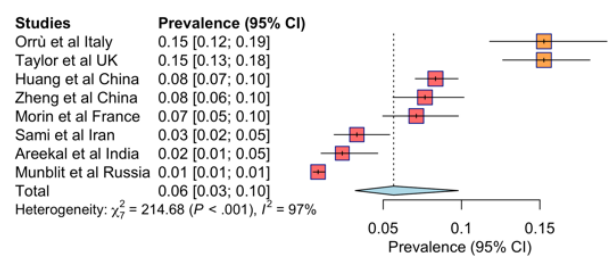

Myalgia

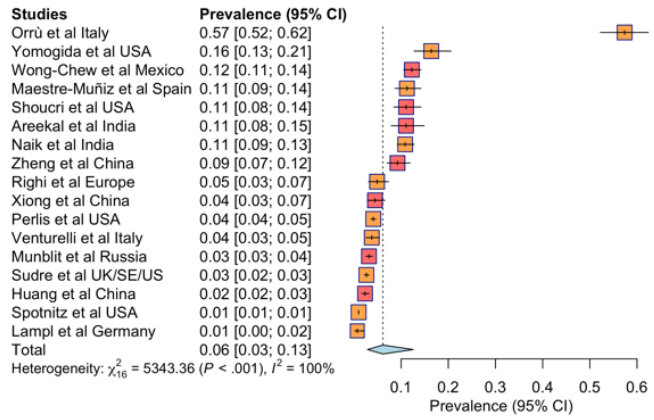


medRxiv preprint doi: https://doi.org/10.1101/2021.11.15.21266377; this version posted November 16, 2021. The copyright holder for this preprint (which was not certified by peer review) is the author/funder, who has granted medRxiv a license to display the preprint in perpetuity.

\section{eFigure 6 Cont'd}

It is made available under a CC-BY 4.0 International license .

\section{Chest pain}

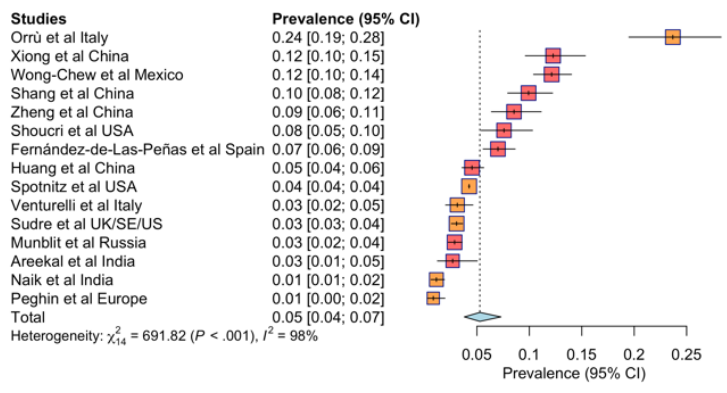

Abdominal pain

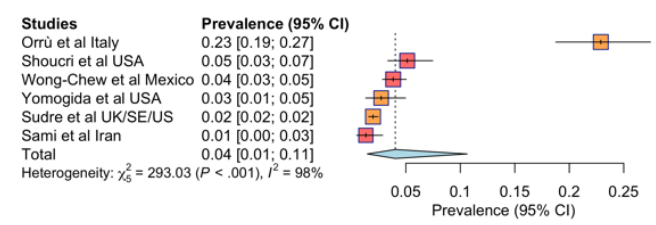

Smell or Taste

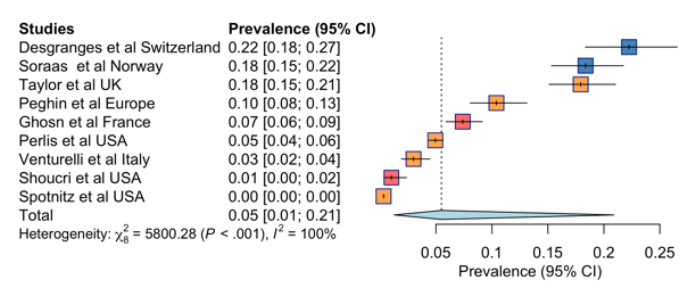

Headache

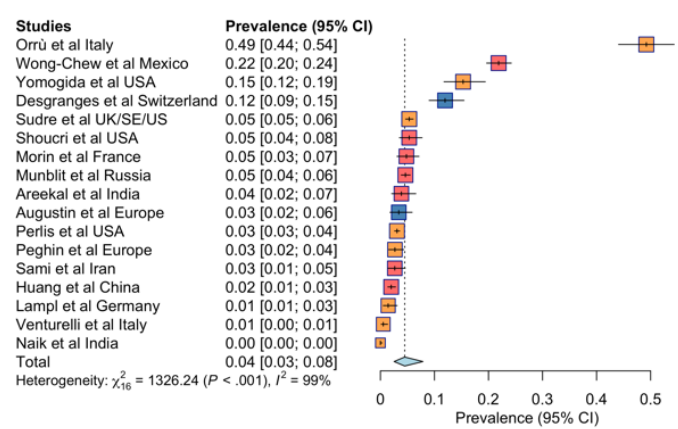


medRxiv preprint doi: https://doi.org/10.1101/2021.11.15.21266377; this version posted November 16, 2021. The copyright holder for this preprint (which was not certified by peer review) is the author/funder, who has granted medRxiv a license to display the preprint in perpetuity.

\section{eFigure 6 Cont'd}

It is made available under a CC-BY 4.0 International license .

Diarrhea

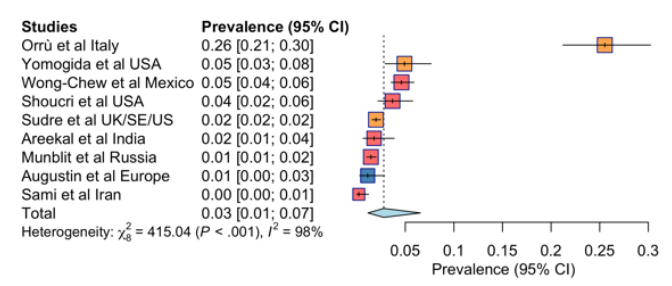

$+2$

Fever
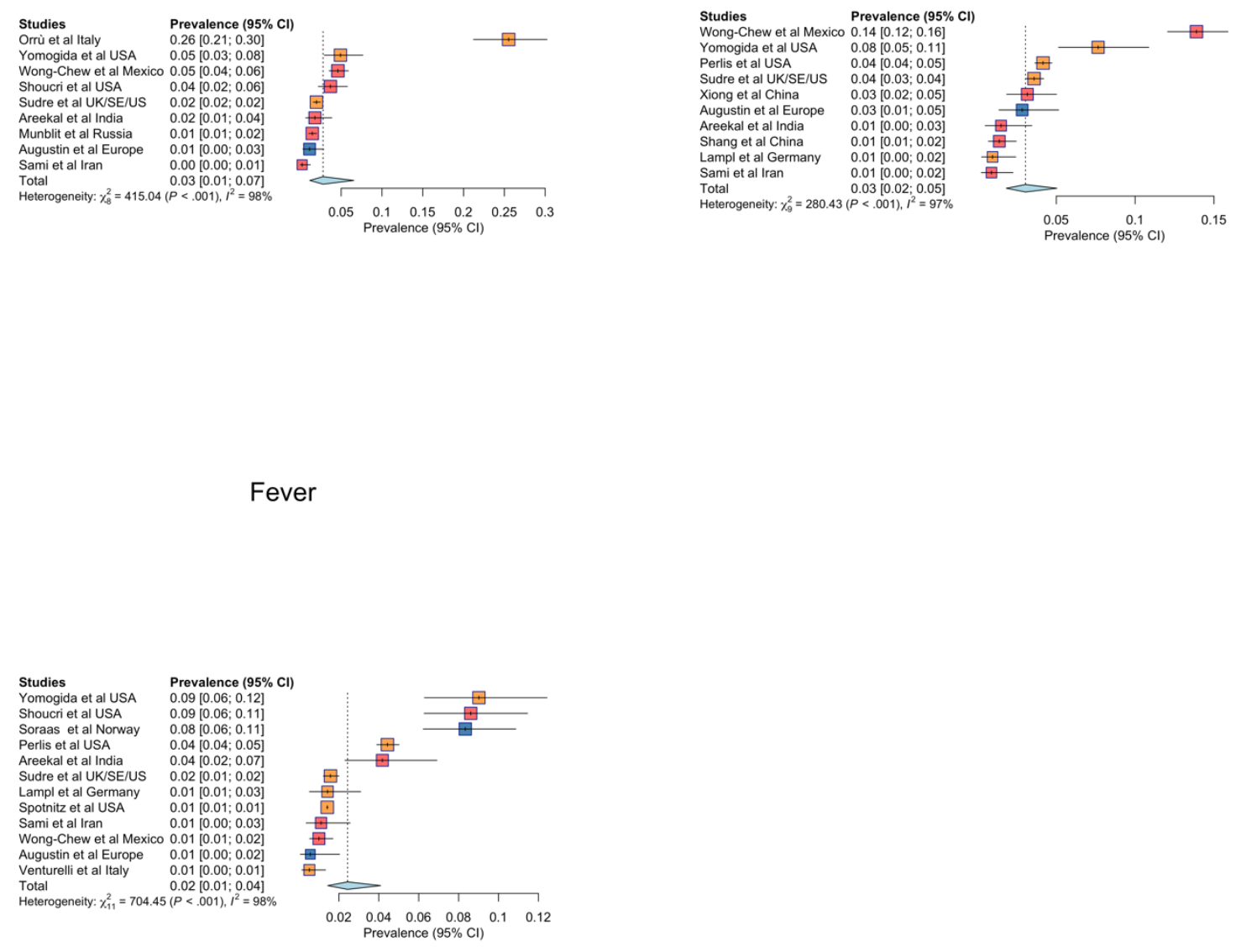

Sore throat

eFigure 6 reports the forest plot of pooled estimated PASC symptoms from the meta-analysis. The legend is consistent across all subfigures where blue, orange, red represents studies with non-hospitalized population, mixed of hospitalized and non-hospitalized population, and hospitalized population respectively.

Study population

$\square$ Not Hospitalized

$\square$ Hospitalized and Non-Hospitalized Mix

$\square$ Hosvitalized 
medRxiv preprint doi: https://doi.org/10.1101/2021.11.15.21266377; this version posted November 16, 2021. The copyright holder for this preprint (which was not certified by peer review) is the author/funder, who has granted medRxiv a license to display the preprint in perpetuity.

It is made available under a CC-BY 4.0 International license .

eFigure 7. Forest plots of risk factors for PASC by a) female and b) asthma

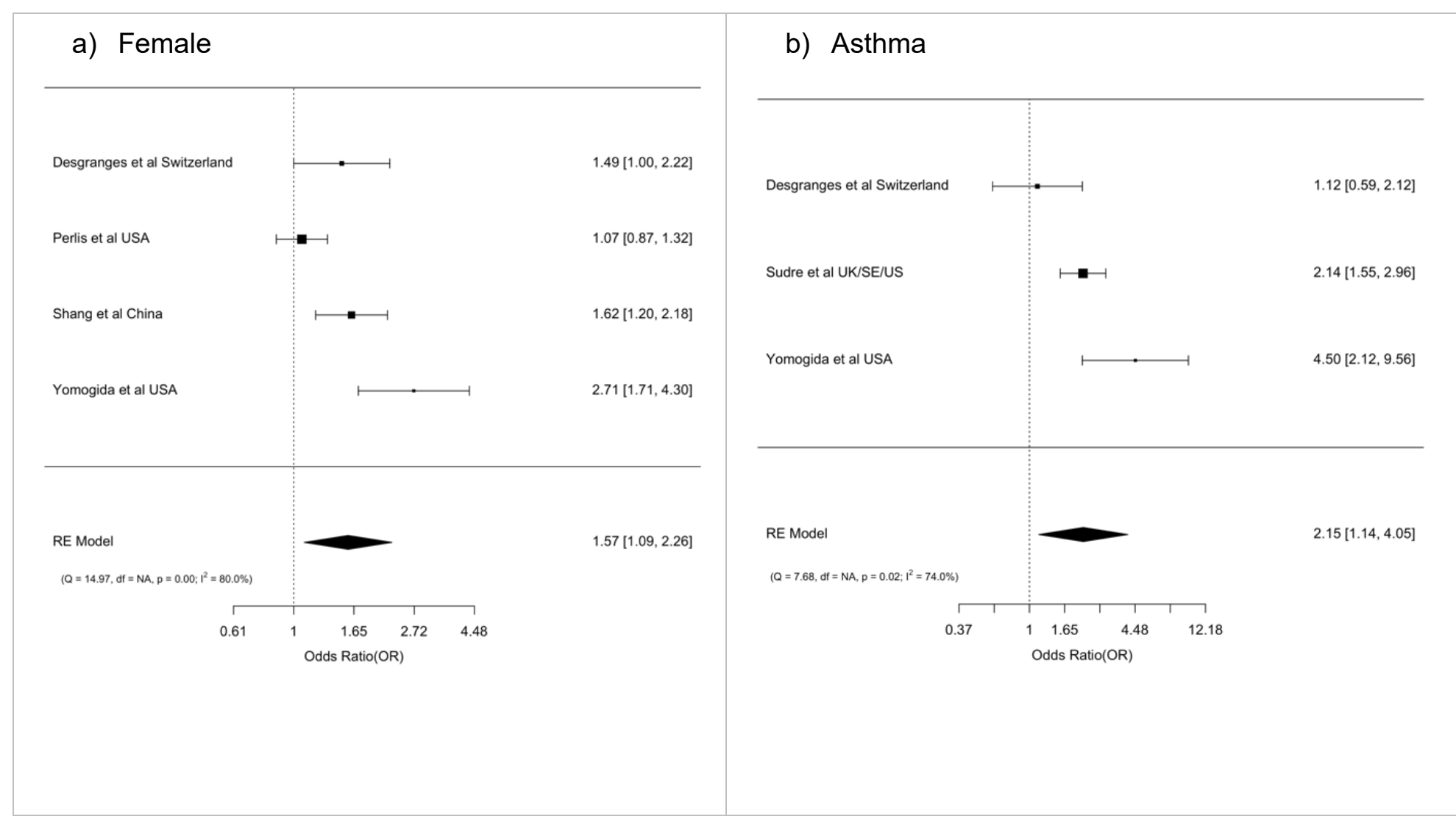

eFigure 7 reports estimated Odds Ratios for female sex and pre-existing asthma as a predictor of PASC. 


\section{eReferences}

1. Knapp G, Biggerstaff BJ, Hartung J. Assessing the Amount of Heterogeneity in RandomEffects Meta-Analysis. Biom J. 2006;48(2):271-285. doi:10.1002/bimj.200510175

2. Onur I, Velamuri M. The gap between self-reported and objective measures of disease status in India. PLOS ONE. 2018;13(8):e0202786. doi:10.1371/journal.pone.0202786

3. Jaffri A, Jaffri UA. Post-Intensive care syndrome and COVID-19: crisis after a crisis? Heart Lung. 2020;49(6):883-884. doi:10.1016/j.hrtlng.2020.06.006

4. Davis HE, Assaf GS, McCorkell L, et al. Characterizing Long COVID in an International Cohort: 7 Months of Symptoms and Their Impact.; 2021:2020.12.24.20248802. doi:10.1101/2020.12.24.20248802

5. Augustin M, Schommers P, Stecher M, et al. Post-COVID syndrome in non-hospitalised patients with COVID-19: a longitudinal prospective cohort study. Lancet Reg Health Eur. 2021;6:100122-100122.

6. Naik S, Soneja M, Haldar S, et al. Post COVID-19 Sequelae: A Prospective Observational Study from Northern India.; 2021:2021.06.28.21259658. doi:10.1101/2021.06.28.21259658

7. Menges D, Ballouz T, Anagnostopoulos A, et al. Burden of Post-COVID-19 Syndrome and Implications for Healthcare Service Planning: A Population-based Cohort Study. Published online 2021.

8. Perlis RH, Green J, Santillana M, et al. Persistence of symptoms up to 10 months following acute COVID-19 illness. MedRxiv Prepr Serv Health Sci. Published online 2021.

9. Wong-Chew RM, Cabrera EXR, Valdez CAR, et al. Symptom Cluster Analysis of long COVID-19 in Patients Discharged from the Temporary COVID-19 Hospital in Mexico City: A Longitudinal Study. Published online June 1, 2021. doi:10.20944/preprints202106.0011.v1

10. Yomogida KS, Zhu S, Rubino F, Figueroa W, Barin N, Holman E. Longitudinal Surveillance of Post-Acute Sequelae of SARS-CoV-2 (PASC) Among Long Beach City Residents, April 1, 2020-December 10, 2020. Published online 2021.

11. Sudre $\mathrm{CH}$, Murray B, Varsavsky $\mathrm{T}$, et al. Attributes and predictors of long COVID. Nat Med. 2021;27(4):626-631. doi:10.1038/s41591-021-01292-y

12. Desgranges F, Tadini E, Munting A, et al. Post-COVID-19 syndrome in outpatients: a cohort study. Published online 2021.

13. Lin L, Chu H, Murad MH, et al. Empirical Comparison of Publication Bias Tests in MetaAnalysis. J Gen Intern Med. 2018;33(8):1260-1267. doi:10.1007/s11606-018-4425-7 